\title{
Generalized Fuzzy Shapley Function for Fuzzy Games
}

\author{
Chunqiao Tan and Xiaohong Chen \\ School of Business, Central South University, Changsha 410083, China \\ Correspondence should be addressed to Chunqiao Tan; chunqiaot@sina.com
}

Received 11 November 2013; Revised 12 June 2014; Accepted 13 June 2014; Published 3 August 2014

Academic Editor: Mehmet Sezer

Copyright (C) 2014 C. Tan and X. Chen. This is an open access article distributed under the Creative Commons Attribution License, which permits unrestricted use, distribution, and reproduction in any medium, provided the original work is properly cited.

\begin{abstract}
A generalized fuzzy Shapley function for fuzzy games is proposed. First, a game with fuzzy characteristic function is introduced. Based on Hukuhara difference, the fuzzy Hukuhara-Shapley function is proposed as a solution concept to this class of fuzzy games. Some of its properties are shown. An equivalent axiomatic characterization of the fuzzy Hukuhara-Shapley function is given. Furthermore, a generalized fuzzy Shapley function for games with fuzzy coalition and fuzzy characteristic function is developed. It is shown that the simplified expression of the generalized fuzzy Shapley function can be regarded as the generalization of the fuzzy Shapley function defined for some particular games with fuzzy coalition and fuzzy characteristic function.
\end{abstract}

\section{Introduction}

The Shapley value [1] is a well-known solution concept in cooperative game theory, which has been investigated by a number of researchers. Most of them treat games with crisp coalitions. However, there are some situations where some agents do not fully participate in a coalition, but to a certain extent. In a class of production games, partial participation in a coalition means offering a part of resources, while full participation means offering all of resources. A coalition including some players who participate partially can be treated as a so-called fuzzy coalition, introduced by Aubin $[2,3]$. It shows to what extent a player transfers his/her representability [4] and is also called a rate of participation. Thus it can describe different participation levels of different players in different game situations, varying from noncooperation to full cooperation.

After the pioneering work by Aubin [3], the Shapley function for games with fuzzy coalition has received more and more attentions. Butnariu [5] gave the expression of the Shapley function on a limited class of fuzzy games with proportional values form. However, most games with proportional values are neither monotone nondecreasing nor continuous with regard to rates of players' participation. In order to overcome this limitation, Tsurumi et al. [6] defined a new Shapley value on a new class of fuzzy games with Choquet integral form. This class of fuzzy games is both monotone nondecreasing and continuous with regard to rates of players' participation. Branzei et al. [7] also introduced a concept of Shapley value for games with fuzzy coalition, which was defined by the associated crisp game corresponding to fuzzy game. Butnariu and Kroupa [8] extend this kind of fuzzy games with proportional values to fuzzy games with weighted function, and the corresponding Shapley function was given. Li and Zhang [9] proposed a simplified expression of the Shapley function for games with fuzzy coalition, which can be regarded as the generalization of Shapley functions defined in some particular games with fuzzy coalition.

On the other hand, by using cooperative game theory, Owen considered linear production programming problems in which multiple decision-makers pool resources to produce some goods [10]. An objective function of the linear production programming problem was represented as total revenue from selling some kinds of goods, and the problem was formulated as a linear programming problem in which, subject to the resource constraints, the revenue is maximized. In many decision-making situations, imprecision and uncertainty are often present due to (a) incomplete information, (b) conflicting evidence, (c) ambiguous information, and (d) subjective information. It can be seen that the possible values of parameters of this kind of production games model are often only imprecisely or ambiguously known to decisionmakers. With this observation in mind, it would be certainly 
more appropriate to interpret the decision-makers' understanding of the parameters as fuzzy numerical data which can be represented by means of fuzzy sets of the real line known as fuzzy numbers. It reflects the decision-makers' ambiguous or fuzzy understanding of the nature of the parameters in the problem-formulation process [11]. The resulting production games problem involving fuzzy parameters would be viewed as a more realistic version than the conventional one $[12,13]$.

From fuzzy mathematical programming perspective, Nishizaki and Sakawa [11] investigated cooperative game problems with fuzzy characteristic functions. Mares [14, 15] and Mares and Vlach [16] were also concerned with the uncertainty in the value of the characteristic function associated with a game, where the characteristic function was expressed by fuzzy number. At the same time, they discussed the fuzzy Shapley values of this kind of fuzzy game. Borkotokey [17] considered a cooperative game with fuzzy coalitions and fuzzy characteristic function simultaneously. A Shapley function in the fuzzy sense was proposed as a solution concept to this class of fuzzy games. Yu and Zhang [18] studied a class of particular games with fuzzy coalitions and a fuzzy characteristic function with Choquet integral form and gave the explicit form of the Shapley value for this class of fuzzy games. However, as Li and Zhang [9] pointed out, there were other many approaches to extend cooperative games to fuzzy games besides Choquet integral method [6] and proportional values method [5]. Which one is more natural? The specific game situation is needed to be considered, because each fuzzy game may only be suitable for a certain case. Therefore, as a kind of special fuzzy game with Choquet integral form, the fuzzy Shapley function of the fuzzy game was only suitable for a certain case. In order to improve this limit, in this paper, we pay more attentions to generalized fuzzy game with fuzzy coalitions and fuzzy characteristic function, and a generalized expression of the fuzzy Shapley function is proposed for the generalized fuzzy game.

In order to do this, this paper is organized as follows. In Section 2, we briefly review some concepts of interval numbers and fuzzy numbers and introduce the Hukuhara difference on interval numbers and fuzzy numbers. In Section 3, a game with fuzzy characteristic functions is introduced, and its fuzzy Hukuhara-Shapley function is proposed, and some of its properties are investigated. Furthermore, an applicable example is given. In Section 4, we investigate a new class of games with fuzzy coalitions and fuzzy characteristic function and give a simplified expression of the generalized fuzzy Shapley function for the new fuzzy games. It is shown that it can be regarded as a generalization of the fuzzy Shapley function defined in the proposed fuzzy games with some particular games with fuzzy coalition and fuzzy characteristic function. Finally, in Section 5, we summarize the main conclusions of the paper.

\section{Preliminaries}

In this section, we start by providing a summary of some concepts of interval numbers and fuzzy numbers, which will be used throughout the paper.
2.1. Interval Numbers. Let $\mathbb{R}$ be $(-\infty, \infty)$, that is, the set of all real numbers. Given $x^{-}, x^{+} \in \mathbb{R}$ and $x^{-} \leq x^{+}$, the closed interval $\left[x^{-}, x^{+}\right]$defines an interval number $\bar{x}=\left[x^{-}, x^{+}\right]=$ $\left\{x \in \mathbb{R} \mid x^{-} \leq x \leq x^{+}\right\}$. Obviously, when $x^{-}=x^{+}$, the interval number $\bar{x}$ reduces to a real number $x^{-}$or $x^{+}$. We say that a real number $x$ is a member of an interval number $\bar{x}$, written as $x \in \bar{x}$, if $x^{-} \leq x \leq x^{+}$. Let us denote by $\mathbb{R}$ the class of all closed and bounded intervals in $\mathbb{R}$. Throughout this paper, when we say that $\bar{x}$ is a closed interval, we implicitly mean that $\bar{x}$ is also bounded in $\mathbb{R}$.

In the following, we will briefly review the order relation and basic operation of interval numbers [19].

Definition 1. For any interval number $\bar{x}$ and $\bar{y}, \bar{x}=\left[x^{-}, x^{+}\right]$, $\bar{y}=\left[y^{-}, y^{+}\right] \in \mathbb{R} \mathbb{R}$.

Consider the following:

(1) $\bar{x} \subseteq \bar{y}$ iff $x^{-} \geq y^{-}, x^{+} \leq y^{+}$,

(2) $\bar{x} \leq \bar{y}$ iff $x^{-} \leq y^{-}, x^{+} \leq y^{+}$,

(3) $\bar{x}=\bar{y}$ iff $x^{-}=y^{-}, x^{+}=y^{+}$.

Definition 2. For any interval numbers $\bar{x}=\left[x^{-}, x^{+}\right], \bar{y}=$ $\left[y^{-}, y^{+}\right] \in \mathbb{R}$, the arithmetic operations on interval numbers are defined as follows:

(1) $\bar{x}+\bar{y}=\left[x^{-}+y^{-}, x^{+}+y^{+}\right]$,

(2) $\bar{x}-\bar{y}=\left[x^{-}-y^{+}, x^{+}-y^{-}\right]$,

(3) $\bar{x} \times \bar{y}=\left[\min \left(x^{-} y^{-}, x^{-} y^{+}, x^{+} y^{-}, x^{+} y^{+}\right), \max \left(x^{-} y^{-}\right.\right.$, $\left.\left.x^{-} y^{+}, x^{+} y^{-}, x^{+} y^{+}\right)\right]$,

(4) $\alpha(\bar{x}+\bar{y})=\alpha \bar{x}+\alpha \bar{y}$.

It is known that scalar multiplication is a special case of interval numbers multiplication operation. Scalar multiplication can be expressed by $\alpha \bar{x}=\left[\alpha x^{-}, \alpha x^{+}\right]$, if $\alpha \geq 0$; $\alpha \bar{x}=\left[\alpha x^{+}, \alpha x^{-}\right]$, if $\alpha<0$.

In general, according to Definition 2 , for $\bar{x}, \bar{y}, \bar{z} \in \mathbb{R}$, $\bar{x}+\bar{y}=\bar{z}$ does not mean that $\bar{x}=\bar{z}-\bar{y}$. For example, $[1,3]-[1,3]=[-2,2] \neq 0$ (unless $\bar{x}=\{x\}$ is a singleton). To overcome this situation, next we introduce $\mathrm{H}$-difference of interval numbers, called Hukuhara difference [20-22], which is denoted by $-_{\mathrm{H}}$ in this paper. Let $\bar{x}=\left[x^{-}, x^{+}\right]$and $\bar{y}=$ $\left[y^{-}, y^{+}\right]$be two closed intervals in $\mathbb{R}$. For a closed interval $\bar{z}=\left[z^{-}, z^{+}\right]$such that $\bar{x}=\bar{y}+\bar{z}, \bar{z}$ is called the Hukuhara difference. Since $\bar{x}=\bar{y}+\bar{z}$, it is easy to see that $x^{-}=y^{-}+z^{-}$ and $x^{+}=y^{+}+z^{+}$; that is, $z^{-}=x^{-}-y^{-}$and $z^{+}=x^{+}-y^{+}$. Therefore, the closed interval $\bar{z}$ exists if $x^{-}-y^{-} \leq x^{+}-y^{+}$. In this case, $\bar{z}=\left[x^{-}-y^{-}, x^{+}-y^{+}\right]$and we also denote $\bar{z}=\bar{x}-{ }_{\mathrm{H}} \bar{y}$. When we say that the Hukuhara difference $\bar{z}=\bar{x}-{ }_{\mathrm{H}} \bar{y}$ exists, we implicitly mean that $x^{-}-y^{-} \leq x^{+}-y^{+}$. An important property of $-_{\mathrm{H}}$ is that $\bar{x}-{ }_{\mathrm{H}} \bar{x}=0$, and if $\bar{x}-{ }_{\mathrm{H}} \bar{y}$ exists, $\mathrm{H}-$ difference is unique [22]. For any positive number $\alpha>0$, $\alpha\left(\bar{x}-_{\mathrm{H}} \bar{y}\right)=\alpha \bar{x}-_{\mathrm{H}} \alpha \bar{y}$. In general, $\bar{x}-\bar{y} \neq \bar{x}-_{\mathrm{H}} \bar{y}$.

Definition 3. For any $i=1,2, \ldots$, let $\bar{a}_{i}=\left[a_{i}^{-}, a_{i}^{+}\right] \in \mathbb{R}$ and define

$$
\sum_{i}^{\oplus} \bar{a}_{i}=\sum_{\bar{a}_{i} \geq 0} \bar{a}_{i}-{ }_{\mathrm{H}} \sum_{\bar{a}_{i} \geq 0}\left(-\bar{a}_{i}\right) .
$$

It is easy to prove the following conclusions. 
Proposition 4. (i) Let $N=\{1,2, \ldots, n\}, \bar{a}_{i}=\left[a_{i}^{-}, a_{i}^{+}\right], N_{1} \cap$ $N_{2}=\varnothing$, and $N_{1} \cup N_{2}=N$; then

$$
\sum_{i \in N}^{\oplus} \bar{a}_{i}=\sum_{i \in N_{1}}^{\oplus} \bar{a}_{i}+\sum_{i \in N_{2}}^{\oplus} \bar{a}_{i} .
$$

(ii) Let $\bar{a}=\left[a^{-}, a^{+}\right] \geq 0$, for any $\lambda_{i} \in \mathbb{R}$; then

$$
\sum_{i}^{\oplus}\left(\lambda_{i} \bar{a}\right)=\left(\sum_{i} \lambda_{i}\right) \bar{a}
$$

2.2. Fuzzy Numbers. A general fuzzy set over a given set (or space) $X$ of elements (the universe) is usually defined by its membership function $m: X \rightarrow T \subseteq[0,1]$ and a fuzzy (sub)set $u$ of $X$ is uniquely characterized by the pairs $\left(x, m_{u}(x)\right)$ for each $x \in X$; the value $m_{u}(x) \in[0,1]$ is the membership grade of $x$ to the fuzzy set $u$ and $m_{u}$ is the membership function of a fuzzy set $u$ over $X[23,24]$ for the origins of fuzzy set theory. The support of $u$ is the (crisp) subset of points of $X$ at which the membership grade $m_{u}(x)$ is positive: $\operatorname{supp}(u)=\left\{x \mid x \in X, m_{u}(x)>0\right\}$. For $\lambda \in[0,1]$, the $\lambda$-level-cut of $u$ (or simply the $\lambda$-cut) is defined by $[u]_{\lambda}=$ $\left\{x \mid x \in X, m_{u}(x) \geq \lambda\right\}$ and for $\lambda=0$ (or $\lambda \rightarrow+0$ ) by the closure of the support $[u]_{0}=\operatorname{cl}\left\{x \mid x \in X, m_{u}(x)>0\right\}$, where $\mathrm{cl}$ denotes the closure of sets.

The following properties characterize the normal, convex, and upper semicontinuous fuzzy sets (in terms of the levelcuts):

(B1) $[u]_{\lambda}$ is the spaces of nonempty compact and compact convex sets of $\mathbb{R}^{n}$ for all $\lambda \in[0,1]$;

(B2) $[u]_{\lambda} \subseteq[u]_{\beta}$ for $\lambda \geq \beta$;

(B3) $[u]_{\lambda}=\bigcap_{k=1}^{\infty}[u]_{\lambda_{k}}$ for all increasing sequences $\lambda_{k} \uparrow \lambda$ converging to $\lambda$.

Furthermore, any family $\left\{U_{\lambda} \mid \lambda \in[0,1]\right\}$ satisfying conditions (B1)-(B3) represents the level-cuts of a fuzzy set $u$ having $[u]_{\lambda}=U_{\lambda}$.

To quantify fuzzy concepts, we use the following fuzzy numbers [25].

Definition 5. A fuzzy number, denoted by $\widetilde{a}$, is a fuzzy subset of $\mathbb{R}$ with membership function $m_{\tilde{a}}: \mathbb{R} \rightarrow[0,1]$ satisfying the following conditions.

(i) There exists at least one number $a_{0} \in \mathbb{R}$ such that $m_{\tilde{a}}\left(a_{0}\right)=1$;

(ii) $m_{\tilde{a}}(x)$ is nondecreasing on $\left(-\infty, a_{0}\right]$ and nonincreasing on $\left[a_{0},+\infty\right)$;

(iii) $m_{\tilde{a}}\left(a_{0}\right)$ is upper semicontinuous; that is, $\lim _{x \rightarrow x_{0^{+}}}$ $m_{\tilde{a}}(x)=m_{\tilde{a}}\left(x_{0}\right)$ if $x_{0}<a_{0}$ and

$$
\lim _{x \rightarrow x_{0^{-}}} m_{\tilde{a}}(x)=m_{\tilde{a}}\left(x_{0}\right) \quad \text { if } x_{0}>a_{0} ;
$$

(iv) $\operatorname{Supp}(\widetilde{a})$ is compact, where $\operatorname{Supp}(\widetilde{a})=\{x \in \mathbb{R} \mid$ $\left.m_{\tilde{a}}(x)>0\right\}$.
An important type of fuzzy numbers in common use is the trapezoidal fuzzy number [21] whose membership function has the form

$$
m_{\tilde{a}}(x)= \begin{cases}1, & \text { if } x \in\left[a_{b}, a_{c}\right], \\ \frac{x-a_{l}}{a_{b}-a_{l}}, & \text { if } x \in\left[a_{l}, a_{b}\right), \\ \frac{x-a_{r}}{a_{c}-a_{r}}, & \text { if } x \in\left(a_{c}, a_{r}\right], \\ 0, & \text { otherwise, }\end{cases}
$$

where $a_{l}, a_{b}, a_{c}, a_{r} \in \mathbb{R}$ with $a_{l} \leq a_{b} \leq a_{c} \leq a_{r}$ and the trapezoidal fuzzy number $\tilde{a}$ is simply denoted by $\left(a_{l}, a_{b}, a_{c}, a_{r}\right)$. It is called nonnegative if $a_{l} \geq 0$. The trapezoidal fuzzy number degenerates to be a triangular fuzzy number when $a_{b}=a_{c}$, while it becomes an interval number (i.e., a rectangular fuzzy) when $a_{l}=a_{b}$ and $a_{c}=a_{r}$. Any crisp real number $a$ can be regarded as a special trapezoidal fuzzy number with $a_{l}=a_{b}=a_{c}=a_{r}=a$. In this paper, the set of all fuzzy numbers on $\mathbb{R}$ is denoted by $\mathbb{E R}$.

Let any fuzzy number $\widetilde{a} \in \mathbb{R} \mathbb{R}$ have membership function $m_{\tilde{a}}$, and the level set (or $\lambda$-cut) is defined as $\widetilde{a}_{\lambda}=\{x \in \mathbb{R} \mid$ $\left.m_{\tilde{a}} \geq \lambda\right\}, \lambda \in[0,1]$. It follows from the properties of the membership function of a fuzzy number $\widetilde{a}$ that each of its $\lambda$ cuts $\widetilde{a}_{\lambda}$ is an interval number, denoted by $\widetilde{a}_{\lambda}=\left[a_{\lambda}^{-}, a_{\lambda}^{+}\right]$.

Definition 6 (see [25]). Let $\widetilde{a}$ be a fuzzy number; $\widetilde{a}=$ $\bigcup_{\lambda \in[0,1]} \lambda \widetilde{a}_{\lambda}$ (decomposition theory), where $\widetilde{a}_{\lambda}=\{x \in \mathbb{R} \mid$ $\left.m_{\tilde{a}} \geq \lambda\right\}=\left[a_{\lambda}^{-}, a_{\lambda}^{+}\right]$is the $\lambda$-cut set of $\widetilde{a}$ for any $\lambda \in[0,1]$. For all $\widetilde{b} \in \mathbb{R} \mathbb{R}$

(i) $\tilde{a} \subset \widetilde{b}$ iff $\tilde{a}_{\lambda} \subset \widetilde{b}_{\lambda}$;

(ii) $\widetilde{a} \leq \widetilde{b}$ iff $\widetilde{a}_{\lambda} \leq \widetilde{b}_{\lambda}$;

(iii) $\widetilde{a}=\widetilde{b}$ iff $\widetilde{a}_{\lambda}=\widetilde{b}_{\lambda}$;

(iv) if $\lambda_{1} \leq \lambda_{2}$, then $\widetilde{a}_{\lambda_{1}} \supseteq \widetilde{a}_{\lambda_{2}}$.

Let $\tilde{a}, \tilde{b} \in \mathbb{R}$, and let $*$ be a binary operation on $\mathbb{R}$. $\lambda$ cuts of the fuzzy number $\widetilde{a} * \widetilde{b}$ can be calculated because of the following:

$$
\begin{gathered}
(\tilde{a}+\tilde{b})_{\lambda}=\tilde{a}_{\lambda}+\widetilde{b}_{\lambda}=\left[a_{\lambda}^{-}+b_{\lambda}^{-}, a_{\lambda}^{+}+b_{\lambda}^{+}\right], \\
(\tilde{a}-\tilde{b})_{\lambda}=\widetilde{a}_{\lambda}-\widetilde{b}_{\lambda}=\left[a_{\lambda}^{-}-b_{\lambda}^{+}, a_{\lambda}^{+}-b_{\lambda}^{-}\right], \\
(m \widetilde{a})_{\lambda}=m \widetilde{a}_{\lambda}=\left[m a_{\lambda}^{-}, m a_{\lambda}^{+}\right], \quad \forall m \in \mathbb{R}, m>0 .
\end{gathered}
$$

Employing the $\lambda$-cut representation, arithmetic operations on fuzzy numbers are defined in terms of the wellestablished arithmetic operations on interval numbers [25].

Definition 7. Given any pair of fuzzy numbers, $\widetilde{a}, \widetilde{b} \in \mathbb{R} R$, the basic operations on the $\lambda$-cuts of $\tilde{a}$ and $\tilde{b}$ are defined for all $\lambda \in[0,1]$ by the general formula

$$
\widetilde{a} * \widetilde{b}=\bigcup_{\lambda \in[0,1]} \lambda\left(\widetilde{a}_{\lambda} * \widetilde{b}_{\lambda}\right) .
$$


However, similar to the interval number operations, in the fuzzy contexts, equation $\widetilde{a}=\widetilde{b}+\widetilde{c}$ is not equivalent to $\widetilde{c}=\widetilde{a}-\widetilde{b}=\widetilde{a}+(-1) \widetilde{b}$ or $\widetilde{b}=\widetilde{a}-\widetilde{c}=\widetilde{a}+(-) \widetilde{c}$. This has motivated the introduction of the following Hukuhara difference [22].

Definition 8. Given $\tilde{a}, \tilde{b} \in \mathbb{R} R$, if there exists $\tilde{c} \in \mathbb{R} \mathbb{R}$ such that $\widetilde{a}=\widetilde{b}+\widetilde{c} \in \mathbb{R}$, then $\widetilde{c}$ is called the Hukuhara difference (H-difference), denoted by $\widetilde{a}-{ }_{H} \widetilde{b}=\widetilde{c}$.

Clearly, $\widetilde{a}-{ }_{\mathrm{H}} \widetilde{a}=0$; if $\widetilde{a}--_{\mathrm{H}} \widetilde{b}$ exists, it is unique. And the $\lambda$-cuts of H-difference are $\left(\widetilde{a}-{ }_{H} \widetilde{b}\right)_{\lambda}=\left[a_{\lambda}^{-}-b_{\lambda}^{-}, a_{\lambda}^{+}-b_{\lambda}^{+}\right]$; and $\alpha\left(\widetilde{a}-{ }_{H} \widetilde{b}\right)=\alpha \widetilde{a}-{ }_{H} \alpha \widetilde{b}$, for $\alpha \in \mathbb{R}, \alpha>0$. The H-difference inverts the addition of fuzzy numbers. But the Hukuhara difference between two fuzzy numbers does not always exist. Regarding the existence of the Hukuhara difference, there is an extensive literature described in the study by Dubois et al. in [21].

Proposition 9. Let $\widetilde{a}, \widetilde{b} \in \mathbb{R R}$. The Hukuhara difference $\widetilde{a}-{ }_{\mathrm{H}} \widetilde{b}$ exists if and only if

$$
\begin{array}{r}
a_{\lambda}^{-}-b_{\lambda}^{-} \leq a_{\beta}^{-}-b_{\beta}^{-} \leq a_{\beta}^{+}-b_{\beta}^{+} \leq a_{\lambda}^{+}-b_{\lambda}^{+}, \\
\forall \lambda, \beta \in(0,1], \beta>\lambda .
\end{array}
$$

Definition 10. Let $X$ be a nonempty set; $P(X)$ denotes its power set. For mapping $H$

$$
\begin{gathered}
H:[0,1] \longrightarrow P(X), \\
\alpha \longmapsto H(\alpha) .
\end{gathered}
$$

If, for any $\alpha_{1}, \alpha_{2} \in[0,1]$, we have

$$
\alpha_{1} \leq \alpha_{2} \Longrightarrow H\left(\alpha_{1}\right) \supseteq H\left(\alpha_{2}\right),
$$

then $H$ is called a nested set of $X$.

Proposition 11. Given $\widetilde{a}, \widetilde{b} \in \mathbb{R} \mathbb{R}$, for any $\lambda \in[0,1]$, let $H(\lambda)=$ $\widetilde{a}_{\lambda}{ }_{\mathrm{H}} \widetilde{b}_{\lambda}=\left[a_{\lambda}^{-}-b_{\lambda}^{-}, a_{\lambda}^{+}-b_{\lambda}^{+}\right]$; then $H(\lambda)$ is a nested set.

Proof. For all $a \in[0,1]$, we have

$$
H(a)=\left[a_{\alpha}^{-}-b_{\alpha}^{-}, a_{\alpha}^{+}-b_{\alpha}^{+}\right]=\left(\tilde{a}-{ }_{\mathrm{H}} \tilde{b}\right)_{a} .
$$

If $\lambda \leq \alpha$, according to Definition 7, we have

$$
\left(\widetilde{A}-{ }_{\mathrm{H}} \widetilde{B}\right)_{\lambda} \supseteq\left(\widetilde{A}-{ }_{\mathrm{H}} \widetilde{B}\right)_{\alpha}, \quad \text { that is, } H(\lambda) \supseteq H(a) .
$$

Thus for any $\lambda \in[0,1], H(\lambda)$ is a nested set.

This completes the proof of Proposition 11.

For $i=1,2, \ldots, \widetilde{a}_{i} \in \mathbb{R}$, we define

$$
\sum_{i}^{\oplus} \widetilde{a}_{i}=\sum_{\widetilde{a}_{i} \geq 0} \widetilde{a}_{i}-\mathrm{H} \sum_{\widetilde{a}_{i} \geq 0}\left(-\widetilde{a}_{i}\right) .
$$

According to representation theorem [25] and Proposition 4 , it is easy to obtain the following conclusions.
Proposition 12. (i) Let $N=\{1,2, \ldots, n\}, \widetilde{a}_{i} \in \mathbb{R}, N_{1} \cap N_{2}=$ $\varnothing$, and $N_{1} \cup N_{2}=N$; then

$$
\sum_{i \in N}^{\oplus} \widetilde{a}_{i}=\sum_{i \in N_{1}}^{\oplus} \widetilde{a}_{i}+\sum_{i \in N_{2}}^{\oplus} \widetilde{a}_{i} .
$$

(ii) Given fuzzy number $\widetilde{a} \geq 0$, for $\lambda_{i} \in \mathbb{R}$, then

$$
\sum_{i}^{\oplus}\left(\lambda_{i} \widetilde{a}\right)=\left(\sum_{i} \lambda_{i}\right) \widetilde{a} .
$$

\section{Fuzzy Hukuhara-Shapley Function for Games with Fuzzy Characteristic Function}

3.1. Crisp Cooperative Games and Shapley Value. A finite transferable utility cooperative game (from now on, simply a game or cooperative game) is a pair $(N, v)$, where $N=$ $\{1,2, \ldots, n\}$ is a finite set of players and $v: P(N) \rightarrow \mathbb{R}^{+}$ is called characteristic function satisfying $v(\varnothing)=0 . P(N)$ is the family of crisp subsets of $N$; that is, $P(N)$ is equivalent to $2^{|N|}$. We will refer to a subset $S$ of $N$ as a coalition or crisp coalition and to $v(S)$ as the worth of $S$, which can be seen as the amount of utility the coalition obtains when the players in $S$ work together. The class of all crisp games with player set $N$ is denoted by $G(N)$. $|S|$ denotes the cardinality of $S$. Given a game $(N, v)$ and a coalition $S$, we write $(N, v)$ for the subgame obtained by restricting $v$ to subsets of $S$ only (i.e., to $\left.2^{|S|}\right)$. If there is no fear of confusion, a (cooperative) game $(N, v)$ is replaced by $v$. For any $A, B \subseteq N, A \cap B=\varnothing$, if $v(A \cup B) \geq v(A)+v(B)$, then $v$ is called superadditive game. The set of all superadditive games is denoted by $G_{0}(N)$. For the sake of simplicity, the bracket is often omitted when a set is written in this paper. For example, we write $i j k$ instead of $\{i, j, k\}$.

As an important solution concept for crisp cooperative games, the Shapley value is defined as follows.

Definition 13. The Shapley value $\varphi_{i}(v)$ of player $i$ with respect to a game $v \in G_{0}(N)$ is a weighted average value of the marginal contribution $v(T \cup i)-v(T)$ of player $i$ alone in all combinations, which is defined by

$$
\varphi_{i}(v)=\sum_{T \subseteq N \backslash i} \frac{(|N|-|T|-1) !|T| !}{|N| !}[v(T \cup i)-v(T)] .
$$

Equation (17) is a unique expression which satisfies three axiomatic characterizations of Shapley value (see the study by Shapley [1]).

Proposition 14 (see [1]). For any $S, S \subseteq N$ and $S \neq \varnothing$, if the $S$-unanimity game $\mu_{s}$ is denoted by

$$
u_{S}(A)= \begin{cases}1, & A \supseteq S, \\ 0, & \text { otherwise, }\end{cases}
$$

then, $\left\{u_{S}\right\}_{S \in 2^{N} \backslash \varnothing}$ is a basis in the linear space $G_{0}(N)$ of all games, and, for $v$, it can be uniquely written as

$$
v=\sum_{S \in 2^{N}, S \neq \varnothing} c_{S}(v) u_{S},
$$


where

$$
c_{S}(v)=\sum_{B \in 2^{N}, B \subseteq S}(-1)^{|S|-|B|} v(B)=\sum_{B \in 2^{N}, B \subseteq S}(-1)^{s-b} v(B),
$$

where $s, b$ denote the cardinality of crisp coalitions $S$ and $B$, respectively.

3.2. Cooperative Games with Fuzzy Characteristic Function. In a crisp cooperative game, characteristic function describes a cooperative game and associates a crisp coalition $S$ with the worth $v(S)$, which is interpreted as the payoff that the coalition $S$ can acquire only through the action of $S$. The cooperative crisp game is based on the assumption that all players and coalitions know the payoff value $v$ before the cooperation begins.

The traditional cooperative game assumes that all data of a game are known exactly by players. However, in real game situations often the players are not able to evaluate exactly some data of the game due to a lack of information or/and imprecision of the available information on the environment or on the behavior of the other players. Taking imprecision of information in decision-making problems into account, this assumption is not realistic because there are many uncertain factors during negotiation and coalition formation. In many situations, the players can have only vague ideas about the real payoff value. Therefore, it is more suitable to incorporate fuzzy characteristic function, represented by fuzzy numbers, into cooperative games. In this section, the fuzzy Hukuhara-Shapley function for games with a fuzzy characteristic function is proposed.

Definition 15. A cooperative game with fuzzy characteristic function form is an ordered pair $(N, \widetilde{v})$ where $\widetilde{v}: P(N) \rightarrow$ $\mathbb{R} \mathbb{R}^{+}=\{\widetilde{a} \in \mathbb{R} \mid \widetilde{a} \geq 0\}$ with $\widetilde{v}(\varnothing)=0$.

Fuzzy characteristic function $\widetilde{v}(S)$ can be interpreted as the maximal fuzzy worth or cost savings that the members of $S$ can obtain when they cooperate. Often we identify the game $(N, \widetilde{v})$ with its fuzzy characteristic function $\widetilde{v}$. The class of games with fuzzy characteristic function is denoted by $F G(N)$. In this paper, we mainly discuss the superadditive games with fuzzy characteristic function; that is, for any two crisp coalitions $S, T \in P(N)$ such that $S \cap T=\varnothing$, for any $\lambda \in(0,1], \widetilde{v}_{\lambda}(S \cup T) \geq \widetilde{v}_{\lambda}(S)+\widetilde{v}_{\lambda}(T)$. Then, for any crisp coalitions $S, T \in P(N), T \subseteq S$ and any $\lambda, \beta \in(0,1]$ such that $\beta>\lambda$, the superadditive game $(N, \widetilde{v})$ also satisfies

$$
\begin{aligned}
v_{\lambda}^{-}(S) & -v_{\lambda}^{-}(T) \leq v_{\beta}^{-}(S)-v_{\beta}^{-}(T) \\
& \leq v_{\beta}^{+}(S)-v_{\beta}^{+}(T) \leq v_{\lambda}^{+}(S)-v_{\lambda}^{+}(T) .
\end{aligned}
$$

According to Proposition 9 it is easy to see that the Hukuhara difference $\widetilde{v}(S)-{ }_{\mathrm{H}} \widetilde{v}(T)$ exists for the superadditive games with fuzzy characteristic function. The set of all superadditive games with fuzzy characteristic functionis denoted by $F G_{0}(N)$.
Definition 16. Given $\widetilde{v} \in F G_{0}(N)$, a carrier of $\widetilde{v}$ is any set $T \subseteq$ $N$ with $\widetilde{v}(S)=\widetilde{v}(S \cap T), \forall S \subseteq N$.

Obviously, players outside any carrier have no influence on the play since they contribute nothing to any coalition. Such a player that does not contribute anything to any coalition is called a null player; that is, for any $T \subseteq N \backslash i$, $\widetilde{v}(T \cup i)=\widetilde{v}(T)$.

Let $(N, \widetilde{v}),(N, \widetilde{w}) \in F G_{0}(N)$; for $\forall S \subseteq N$ define $\widetilde{v}+\widetilde{w}:(\widetilde{v}+$ $\widetilde{w})(S)=\widetilde{v}(S)+\widetilde{w}(S)$. It is easily seen that $(N, \widetilde{v}+\widetilde{w}) \in F G_{0}(N)$.

Definition 17. Let $N=\{1,2, \ldots, n\}$; for any $\widetilde{v} \in F G_{0}(N)$, a function $\widetilde{\varphi}: F G_{0}(N) \rightarrow\left(\mathbb{P} \mathbb{R}^{+}\right)^{n}$, where $\widetilde{\varphi}=\left(\widetilde{\varphi}_{1}, \ldots, \widetilde{\varphi}_{n}\right)$, is said to be a fuzzy Hukuhara-Shapley function on $F G_{0}(N)$ if it satisfies the following three axioms.

Axiom $A_{1}$. If $\widetilde{v} \in F G_{0}(N)$ and $T \subseteq N$ is any carrier of $\widetilde{v}$, then

$$
\sum_{i \in T} \widetilde{\varphi}_{i}(\widetilde{v})=\widetilde{v}(T)
$$

Axiom $A_{2}$. If $\widetilde{v} \in F G_{0}(N)$ and $\pi$ is a permutation of the player set, that is, $\pi: N \rightarrow N$, then, for any $i \in N$,

$$
\widetilde{\varphi}_{\pi i}(\pi \widetilde{v})=\widetilde{\varphi}_{i}(\widetilde{v}) \text {. }
$$

Axiom $A_{3}$. If $\widetilde{v}, \widetilde{w} \in F G_{0}(N)$, then

$$
\widetilde{\varphi}_{i}(\widetilde{v}+\widetilde{w})=\widetilde{\varphi}_{i}(\widetilde{v})+\widetilde{\varphi}_{i}(\widetilde{w}) \quad \forall i \in N
$$

It is easy to obtain the following conclusion.

Proposition 18. If $\widetilde{v} \in F G_{0}(N)$, for any $\tilde{a} \in \mathbb{R}^{+}$, then $(N, \tilde{a} \widetilde{v}) \in F G_{0}(N)$.

Lemma 19. For any $\tilde{a} \in \mathbb{R}^{+}, \tilde{a} \geq 0, S \subseteq N, S \neq \varnothing$, fuzzy Hukuhara-Shapley value of $\tilde{a} u_{S}$ is

$$
\widetilde{\varphi}_{i}\left(\tilde{a} u_{S}\right)= \begin{cases}0, & i \notin S \\ \frac{\tilde{a}}{|S|}, & i \in S .\end{cases}
$$

Proof. It is obvious that $S$ is a carrier of $\tilde{a} u_{S}$. For $i \in N \backslash S$, since $S$ and $S \cup i$ are also carriers of $\widetilde{a} u_{S}$, according to Axiom $\mathrm{A}_{1}$, we have

$$
\begin{aligned}
\sum_{j \in S} \widetilde{\varphi}_{j}\left(\tilde{a} u_{S}\right)=\tilde{a} u_{S}(S)=\tilde{a} u_{S}(S \cup i) \\
=\sum_{j \in S \cup i} \widetilde{\varphi}_{j}\left(\widetilde{a} u_{S}\right)=\sum_{j \in S} \widetilde{\varphi}_{j}\left(\widetilde{a} u_{S}\right)+\widetilde{\varphi}_{i}\left(\widetilde{a} u_{S}\right) .
\end{aligned}
$$

So

$$
\widetilde{\varphi}_{i}\left(\widetilde{a} u_{S}\right)=\sum_{j \in S} \widetilde{\varphi}_{j}\left(\widetilde{a} u_{S}\right)-_{H} \sum_{j \in S} \widetilde{\varphi}_{j}\left(\widetilde{a} u_{S}\right)=0
$$

For $i, j \in S$ and $i \neq j$, let $\pi$ be a permutation of $N$ such that $\pi(i)=j, \pi(j)=i, \pi(k)=k, k=N \backslash\{i, j\}$. First, we prove that, for any $T \subseteq N$,

$$
\widetilde{a} u_{S}(\pi T)=\widetilde{a} u_{S}(T) .
$$


For $T \supseteq S, \pi T \supseteq \pi S=S$. We have

$$
\tilde{a} u_{S}(\pi T)=\tilde{a}=\widetilde{a} u_{S}(T)
$$

For $T \nsupseteq S$, if $i \notin T$, then $j \notin \pi T$. If $j \notin T$, then $i \notin \pi T$. If $k \notin T$ and $k \in S \backslash\{i, j\}$, then $k \notin \pi T$. So $\pi T \subset S$.

Therefore,

$$
\tilde{a} u_{S}(\pi T)=0=\widetilde{a} u_{S}(T)
$$

According to Axiom $\mathrm{A}_{2}$, we have

$$
\widetilde{\varphi}_{j}\left(\widetilde{a} u_{S}\right)=\widetilde{\varphi}_{\pi(i)}\left(\pi\left(\widetilde{a} u_{S}\right)\right)=\widetilde{\varphi}_{i}\left(\widetilde{a} u_{S}\right)
$$
that

According to the above analysis and Axiom $\mathrm{A}_{1}$, we obtain

$$
\sum_{i \in S} \widetilde{\varphi}_{i}\left(\widetilde{a} u_{S}\right)=|S| \widetilde{\varphi}_{i}\left(\widetilde{a} u_{S}\right)=\widetilde{a} u_{S}(S)=\widetilde{a}, \quad \forall i \in S
$$

That is,

$$
\widetilde{\varphi}_{i}\left(\widetilde{a} u_{S}\right)=\frac{\tilde{a}}{|S|}, \quad \forall i \in S .
$$

This completes the proof of Lemma 19.

Lemma 20. If $\widetilde{v} \in F G(N)$, for any $S, \varnothing \neq S \subseteq N$, then $\widetilde{v}$ is a linear combination of $u_{S}$ :

$$
\widetilde{v}(T)=\sum_{S \subseteq Q}^{\oplus} \widetilde{c}_{S}(\widetilde{v}) u_{S}(T), \quad \forall T \subseteq N
$$

where $Q$ is a carrier of $\widetilde{v}$. The coefficients $\widetilde{c}_{S}$ are independent of $Q$ and are given by

$$
\widetilde{\mathcal{c}}_{S}(\widetilde{v})=\sum_{R \subseteq S}^{\oplus}(-1)^{|S|-|R|} \widetilde{v}(R)=\sum_{R \subseteq S}^{\oplus}(-1)^{S-r} \widetilde{v}(R),
$$

where $s, r$ denote the cardinality of coalitions $S$ and $R$, respectively.

Proof. If $T \subseteq Q$, then

$$
\begin{aligned}
\sum_{S \subseteq Q}^{\oplus} \widetilde{c}_{S}(\widetilde{v}) u_{S}(T) & \\
= & \sum_{S \subseteq Q}^{\oplus} \sum_{R \subseteq S}^{\oplus}(-1)^{S-r} \widetilde{v}(R) u_{S}(T) \\
= & \sum_{S \subseteq Q}^{\oplus} \sum_{T \subseteq S}^{\oplus \subseteq S}(-1)^{S-r} \widetilde{v}(R) u_{S}(T) \\
& +\sum_{S \subseteq Q}^{\oplus} \sum_{R \subseteq S}^{\oplus}(-1)^{S-r} \widetilde{v}(R) u_{S}(T) \\
= & \sum_{S \subseteq Q}^{\oplus} \sum_{R \subseteq S}^{\oplus}(-1)^{S-r} \widetilde{v}(R)
\end{aligned}
$$

$$
\begin{aligned}
& =\sum_{S \subseteq T}^{\oplus} \sum_{R \subseteq S}^{\oplus}(-1)^{S-r} \widetilde{v}(R) \quad(\text { since } T \subseteq Q) \\
& =\sum_{R \subseteq T}^{\oplus}\left[\sum_{\substack{R \subseteq S \\
S \subseteq T}}^{\oplus}(-1)^{s-r}\right] \widetilde{v}(R) \\
& =\sum_{R \subseteq T}^{\oplus}\left[\sum_{s=r}^{t}(-1)^{s-r}\left(\begin{array}{l}
t-r \\
s-r
\end{array}\right)\right] \widetilde{v}(R) \quad(\text { let } k=s-r) \\
& =\sum_{R \subseteq T}^{\oplus}\left[\sum_{k=0}^{t-r}(-1)^{k}\left(\begin{array}{c}
t-r \\
k
\end{array}\right)\right] \widetilde{v}(R) \\
& =\sum_{\substack{R \subseteq T \\
R=T}}^{\oplus}\left[\sum_{k=0}^{t-r}(-1)^{k}\left(\begin{array}{c}
t-r \\
k
\end{array}\right)\right] \widetilde{v}(R) \\
& +\sum_{\substack{R \subseteq T \\
R \neq T}}^{\oplus}\left[\sum_{k=0}^{t-r}(-1)^{k}\left(\begin{array}{c}
t-r \\
k
\end{array}\right)\right] \widetilde{v}(R) \\
& =\sum_{\substack{R \subseteq T \\
R=T}}^{\oplus} \widetilde{v}(R)+\sum_{\substack{R \subseteq T \\
R \neq T}}^{\oplus}(1-1)^{t-r} \widetilde{v}(R)=\widetilde{v}(T) .
\end{aligned}
$$

In general, according to the definition of carrier, we have

$$
\begin{aligned}
\widetilde{v}(T) & =\widetilde{v}(T \cap Q)=\sum_{\substack{S \subseteq Q \\
S \neq \varnothing}}{ }^{\oplus} \widetilde{c}_{S}(\widetilde{v}) u_{S}(T \cap Q) \\
& =\sum_{\substack{S \subseteq Q \\
S \neq \varnothing}}{ }^{\oplus} \widetilde{c}_{S}(\widetilde{v}) u_{S}(T) .
\end{aligned}
$$

This completes the proof of Lemma 20.

Remark 21. For $\widetilde{v} \in F G_{0}(N)$ and any $\lambda \in(0,1]$, it is obvious that crisp games $v_{\lambda}^{-}, v_{\lambda}^{+} \in G_{0}(N)$. Assume

$$
\begin{aligned}
& \mathcal{c}_{T}\left(v_{\lambda}^{+}\right)=\sum_{B \in P(N), B \subseteq T}(-1)^{|T|-|B|} \cdot v_{\lambda}^{+}(B), \\
& \mathcal{c}_{T}\left(v_{\lambda}^{-}\right)=\sum_{B \in P(N), B \subseteq T}(-1)^{|T|-|B|} \cdot v_{\lambda}^{-}(B) .
\end{aligned}
$$

According to Proposition 14 , it is seen that $v_{\lambda}^{-}$and $v_{\lambda}^{+}$can be uniquely written as

$$
\begin{aligned}
& v_{\lambda}^{+}(B)=\sum_{T \in P(N), T \neq \varnothing} c_{T}\left(v_{\lambda}^{+}\right) \cdot u_{T}(B), \\
& v_{\lambda}^{-}(B)=\sum_{T \in P(N), T \neq \varnothing} c_{T}\left(v_{\lambda}^{-}\right) \cdot u_{T}(B) .
\end{aligned}
$$

Obviously, $\sum_{T \in P(N), T \neq \varnothing}\left(c_{T}\left(v_{\lambda}^{+}\right)-c_{T}\left(v_{\lambda}^{-}\right)\right) \cdot u_{T}(B)=v_{\lambda}^{+}(B)-$ $v_{\lambda}^{-}(B) \geq 0$. According to representation theorem of fuzzy set [25], it is easy to obtain the same conclusion as Lemma 20. 
Proposition 22. If $\widetilde{v}, \widetilde{w}$ and $\left(\widetilde{v}-{ }_{\mathrm{H}} \widetilde{w}\right) \in F G_{0}(N)$, then

$$
\widetilde{\varphi}_{i}\left(\widetilde{v}-{ }_{\mathrm{H}} \widetilde{w}\right)=\widetilde{\varphi}_{i}(\widetilde{v})-_{\mathrm{H}} \widetilde{\varphi}_{i}(\widetilde{w}) .
$$

Proof. Let $\widetilde{z}=\widetilde{v}-{ }_{\mathrm{H}} \widetilde{w}$; then $\widetilde{v}=\widetilde{w}+\widetilde{z}$. According to Axiom $\mathrm{A}_{3}$, we have

$$
\widetilde{\varphi}_{i}(\widetilde{v})=\widetilde{\varphi}_{i}(\widetilde{w})+\widetilde{\varphi}_{i}(\widetilde{z}) .
$$

So $\widetilde{\varphi}_{i}(\widetilde{z})=\widetilde{\varphi}_{i}(\widetilde{v})-_{\mathrm{H}} \widetilde{\varphi}_{i}(\widetilde{w})$; that is, $\widetilde{\varphi}_{i}\left(\widetilde{v}-{ }_{\mathrm{H}} \widetilde{w}\right)=$ $\widetilde{\varphi}_{i}(\widetilde{v})-{ }_{\mathrm{H}} \widetilde{\varphi}_{i}(\widetilde{w})$.

This completes the proof of Proposition 22.

From Proposition 18, if $\widetilde{c}_{S}(\widetilde{v}) \geq 0$, then $\widetilde{c}_{S}(\widetilde{v}) u_{S} \in F G_{0}(N)$; otherwise, $\left(-c_{S}(\widetilde{v})\right) u_{S} \in F G_{0}(N)$.

Lemma 23. If $\widetilde{v} \in F G_{0}(N)$, then

$$
\widetilde{\varphi}_{i}(\widetilde{v})=\sum_{i \in S \subseteq N} \oplus \frac{\widetilde{c}_{S}(\widetilde{v})}{|S|} .
$$

Proof. According to Lemmas 19 and 20 and Axiom $\mathrm{A}_{3}$, we have

$$
\begin{aligned}
& \widetilde{\varphi}_{i}(\widetilde{v})=\widetilde{\varphi}_{i}\left(\sum_{S \subseteq N}^{\oplus}{\widetilde{c_{S}}}_{S}(\widetilde{v}) u_{S}\right) \\
& =\widetilde{\varphi}_{i}\left(\sum_{\substack{S \subseteq N \\
\widetilde{c}_{S}(\widetilde{v}) \geq 0}} \widetilde{c}_{S}(\widetilde{v}) u_{S}-{ }_{H} \sum_{\substack{S \subseteq N \\
\widetilde{c}_{S}(\widetilde{v}) \pm 0}}\left(-\widetilde{c}_{S}(\widetilde{v})\right) u_{S}\right) \\
& =\widetilde{\varphi}_{i}\left(\sum_{\substack{S \subseteq N \\
\tilde{\mathcal{S}}_{S}(\widetilde{v}) \geq 0}} \widetilde{c}_{S}(\widetilde{v}) u_{S}\right)-{ }_{\mathrm{H}} \widetilde{\varphi}_{i}\left(\sum_{\substack{S \subseteq N \\
\tilde{c}_{S}(\widetilde{v}) \pm 0}}\left(-\widetilde{c}_{S}(\widetilde{v})\right) u_{S}\right) \\
& =\sum_{\substack{S \subseteq N \\
\widetilde{c}_{S}(\widetilde{v}) \geq 0}} \widetilde{\varphi}_{i}\left(\widetilde{c}_{S}(\widetilde{v}) u_{S}\right)-{ }_{\mathrm{H}} \sum_{\substack{S \subseteq N \\
\widetilde{\tau_{S}}(\widetilde{v}) \pm 0}} \widetilde{\varphi}_{i}\left(\left(-\widetilde{c}_{S}(\widetilde{v})\right) u_{S}\right) \\
& =\sum_{\substack{S \subseteq N, i \in S \\
\widetilde{\mathcal{S}_{S}}(\widetilde{v}) \geq 0}} \widetilde{\varphi}_{i}\left(\widetilde{c}_{S}(\widetilde{v}) u_{S}\right)+\sum_{\substack{S \subseteq N, i \notin S \\
\widetilde{c_{S}}(\widetilde{v}) \geq 0}} \widetilde{\varphi}_{i}\left(\widetilde{c}_{S}(\widetilde{v}) u_{S}\right)-_{\mathrm{H}} \\
& \times \sum_{\substack{S \subseteq N, i \in S \\
\widetilde{c}_{S}(\widetilde{v}) \pm 0}} \widetilde{\varphi}_{i}\left(\left(-\widetilde{c}_{S}(\widetilde{v})\right) u_{S}\right)-{ }_{\mathrm{H}} \sum_{\substack{S \subseteq N, i \notin S \\
\widetilde{c_{S}}(\widetilde{v}) \pm 0}} \widetilde{\varphi}_{i}\left(\left(-\widetilde{c}_{S}(\widetilde{v})\right) u_{S}\right) \\
& =\sum_{\substack{S \subseteq N, i \in S \\
\widetilde{c}_{S}(\widetilde{v}) \geq 0}} \widetilde{\varphi}_{i}\left(\widetilde{c}_{S}(\widetilde{v}) u_{S}\right)-{ }_{H} \sum_{\substack{S \subseteq N, i \in S \\
c_{S}(\widetilde{v}) \pm 0}} \widetilde{\varphi}_{i}\left(\left(-\widetilde{c}_{S}(\widetilde{v})\right) u_{S}\right) \\
& =\sum_{\substack{S \subseteq N, i \in S \\
\widetilde{\mathcal{S}_{S}}(\widetilde{v}) \geq 0}} \frac{\widetilde{c}_{S}(\widetilde{v})}{|S|}-\sum_{\substack{S \subseteq N, i \in S \\
\widetilde{\tau_{S}}(\widetilde{v}) \pm 0}} \frac{-\widetilde{c_{S}}(\widetilde{v})}{|S|} \\
& =\sum_{\substack{S \subseteq N \\
i \in S}} \frac{\widetilde{c}_{S}(\widetilde{v})}{|S|}
\end{aligned}
$$

This completes the proof of Lemma 23.
Theorem 24. Given $\widetilde{v} \in F G_{0}(N)$, there exists a unique fuzzy Hukuhara-Shapley function $\widetilde{\varphi}(\widetilde{v})$, satisfying Axioms $A_{1}-A_{3}$, with element

$$
\begin{array}{r}
\widetilde{\varphi}_{i}(\widetilde{v})=\sum_{R \subseteq N \backslash i} \frac{r !(n-r-1) !}{n !}\left[\widetilde{v}(R \cup i)-_{\mathrm{H}} \widetilde{v}(R)\right], \\
i=1,2, \ldots, n,
\end{array}
$$

where $r, n$ denote the cardinality of crisp coalitions $R$ and $N$, respectively.

Proof. According to Lemmas 23 and 19, we have

$$
\begin{aligned}
& \widetilde{\varphi}_{i}(\widetilde{v})=\sum_{i \in S \subseteq N} \oplus \frac{\widetilde{c}_{S}(\widetilde{v})}{|S|}=\sum_{i \in S \subseteq N} \oplus \sum_{R \subseteq S}^{\oplus} \frac{(-1)^{s-r} \widetilde{v}(R)}{s} \\
& =\sum_{R \subseteq N} \oplus\left(\sum_{\substack{R \subseteq S \subseteq N \\
R \cup i \subseteq S}} \oplus \frac{(-1)^{s-r}}{s}\right) \widetilde{v}(R) \\
& =\sum_{\substack{R \subseteq N \\
i \in R}} \oplus\left(\sum_{\substack{R \subseteq S \subseteq N \\
R \cup i \subseteq S}} \oplus \frac{(-1)^{s-r}}{s}\right) \widetilde{v}(R) \\
& +\sum_{\substack{R \subseteq N \\
i \notin R}}^{\oplus}\left(\sum_{\substack{R \subseteq S \subseteq N \\
R \cup i \subseteq S}} \oplus \frac{(-1)^{s-r}}{s}\right) \widetilde{v}(R) \\
& =\sum_{\substack{R \subseteq N \\
i \notin R}} \oplus\left(\sum_{\substack{R \subseteq S \subseteq N \\
R \cup i \subseteq S}} \frac{(-1)^{s-(r+1)}}{s}\right) \widetilde{v}(R \cup i) \\
& +\sum_{\substack{R \subseteq N \\
i \notin R}} \oplus\left(\sum_{\substack{R \subseteq S \subseteq N \\
R \cup i \subseteq S}} \oplus \frac{(-1)^{s-r}}{s}\right) \widetilde{v}(R) \\
& =\sum_{\substack{R \subset N \\
i \notin R}}^{\oplus}\left(\sum_{s=r+1}^{n} \frac{(-1)^{s-(r+1)}\left(\begin{array}{c}
n-r-1 \\
s-r-1
\end{array}\right)}{s}\right) \widetilde{v}(R \cup i) \\
& +\sum_{\substack{R \subseteq N \\
i \notin R}}^{\oplus}\left(\sum_{s=r+1}^{n} \frac{(-1)^{s-r}\left(\begin{array}{c}
n-r-1 \\
s-r-1
\end{array}\right)}{s}\right) \widetilde{v}(R) .
\end{aligned}
$$

Since

$$
\begin{gathered}
\sum_{s=r+1}^{n} \frac{(-1)^{s-r-1}}{s}\left(\begin{array}{c}
n-r-1 \\
s-r-1
\end{array}\right)=\frac{r !(n-r-1) !}{n !}, \\
\sum_{s=r+1}^{n} \frac{(-1)^{s-r}\left(\begin{array}{c}
n-r-1 \\
s-r-1
\end{array}\right)}{s}=-\frac{r !(n-r-1) !}{n !},
\end{gathered}
$$


Therefore,

$$
\begin{aligned}
\widetilde{\varphi}_{i}(\widetilde{v})= & \sum_{\substack{R \subseteq N \\
i \notin R}} \frac{r !(n-r-1) !}{n !} \widetilde{v}(R \cup i) \\
& +\sum_{\substack{R \subseteq N \\
i \notin R}}^{\oplus} \frac{-r !(n-r-1) !}{n !} \widetilde{v}(R) \\
= & \sum_{\substack{R \subseteq N \\
i \notin R}} \frac{r !(n-r-1) !}{n !} \widetilde{v}(R \cup i)-_{\mathrm{H}} \\
& \times \sum_{\substack{R \subseteq N \\
i \notin R}} \frac{r !(n-r-1) !}{n !} \widetilde{v}(R) \quad\left(\text { since } \frac{r !(n-r-1) !}{n !}>0\right) \\
= & \sum_{R \subseteq N \backslash i} \frac{r !(n-r-1) !}{n !}\left[\widetilde{v}(R \cup i)-{ }_{\mathrm{H}} \widetilde{v}(R)\right] .
\end{aligned}
$$

From Lemma 20, it is known that $\widetilde{c}_{S}(\widetilde{v})$ is well defined by $S$ and $\widetilde{v}$. Thus according to Lemma $23, \widetilde{\varphi}_{i}(\widetilde{v})$ is well defined by $\widetilde{v}, N$, and $i$; that is, $\widetilde{\varphi}_{i}(\widetilde{v})$ is well defined by $\widetilde{v}$ and $N$.

In the following, we prove that the function defined by (44) satisfies Axioms $A_{1}-A_{3}$ in Definition 17.

According to Lemmas 19 and 20, it is easy to verify that $\widetilde{\varphi}_{i}(\widetilde{v})$ satisfies Axioms $A_{1}$ and $A_{2}$.

Let $\bar{v}, \bar{w} \in F G_{0}(N)$ and $\bar{v}+\bar{w} \in F G_{0}(N)$; then

$$
\begin{aligned}
\widetilde{\varphi}_{i}(\widetilde{v}+\widetilde{w}) & \\
= & \sum_{R \subseteq N \backslash i} \frac{r !(n-r-1) !}{n !}\left[(\widetilde{v}+\widetilde{w})(R \cup i)-_{\mathrm{H}}(\widetilde{v}+\widetilde{w})(R)\right] \\
= & \sum_{R \subseteq N \backslash i} \frac{r !(n-r-1) !}{n !}\left[(\widetilde{v}(R \cup i)+\widetilde{w}(R \cup i))-_{\mathrm{H}}\right. \\
= & \quad \times(\widetilde{v}(R)+\widetilde{w}(R))] \\
& \sum_{R \subseteq N \backslash i} \frac{r !(n-r-1) !}{n !}\left[\left(\widetilde{v}(R \cup i)-_{\mathrm{H}} \widetilde{v}(R)\right)\right. \\
= & \sum_{R \subseteq N \backslash i} \frac{r !(n-r-1) !}{n !}\left[\widetilde{v}(R \cup i)-{ }_{\mathrm{H}} \widetilde{v}(R)\right] \\
& +\sum_{R \subseteq N \backslash i} \frac{r !(n-r-1) !}{n !}\left[\left(\widetilde{w}(R \cup i){ }_{\mathrm{H}} \widetilde{w}(R)\right)\right] \\
= & \widetilde{\varphi}_{i}(\widetilde{v})+\widetilde{\varphi} \widetilde{\varphi}_{i}(\widetilde{w}) .
\end{aligned}
$$

These complete the proof of Proposition 14.
Remark 25. Given $\widetilde{v} \in F G_{0}(N)$, from Theorem 24, for any $\lambda \in(0,1]$ and $\widetilde{v}_{\lambda}$, there exists a unique interval HukuharaShapley function $\bar{\varphi}\left(\widetilde{v}_{\lambda}\right)$ with element

$$
\begin{array}{r}
\bar{\varphi}_{i}\left(\widetilde{v}_{\lambda}\right)=\sum_{R \subseteq N \backslash i} \frac{r !(n-r-1) !}{n !}\left[\widetilde{v}_{\lambda}(R \cup i)-{ }_{\mathrm{H}} \widetilde{v}_{\lambda}(R)\right], \\
i=1,2, \ldots .
\end{array}
$$

For the interval Hukuhara-Shapley function $\bar{\varphi}\left(\widetilde{v}_{\lambda}\right), \lambda \in$ $(0,1]$, and it has the following properties.

Proposition 26. (i) For any pair $\left(\lambda_{1}, \lambda_{2}\right)$ satisfying $0 \leq \lambda_{1}<$ $\lambda_{2} \leq 1, \bar{\varphi}_{i}\left(\widetilde{v}_{\lambda_{1}}\right) \supseteq \bar{\varphi}_{i}\left(\widetilde{v}_{\lambda_{2}}\right)$.

(ii) For any $\lambda \in(0,1], \bigcap_{\lambda<\lambda^{*}} \bar{\varphi}_{i}\left(\widetilde{v}_{\lambda}\right)=\bar{\varphi}_{i}\left(\widetilde{v}_{\lambda^{*}}\right)$.

Proof. (i) According to (49), it is easy to prove the conclusion.

(ii) Let $\gamma_{R}=r !(n-r-1) ! / n !$; from (49), for any $\lambda^{*} \in(0,1]$, we have

$$
\begin{aligned}
& \bigcap_{\lambda<\lambda^{*}} \bar{\varphi}_{i}\left(\widetilde{v}_{\lambda}\right)=\bigcap_{\lambda<\lambda^{*}} \sum_{R \subseteq N \backslash i} \gamma_{R}\left[\widetilde{v}_{\lambda}(R \cup i)-{ }_{\mathrm{H}} \widetilde{v}_{\lambda}(R)\right] \\
& =\bigcap_{\lambda<\lambda^{*}}\left[\sum_{R \subseteq N \backslash i} \gamma_{R}\left[v_{\lambda}^{-}(R \cup i)-v_{\lambda}^{-}(R)\right]\right. \text {, } \\
& \left.\sum_{R \subseteq N \backslash i} \gamma_{R}\left[v_{\lambda}^{+}(R \cup i)-v_{\lambda}^{+}(R)\right]\right] \\
& =\left[\lim _{\lambda \rightarrow \lambda^{*}} \sum_{R \subseteq N \backslash i} \gamma_{R}\left[v_{\lambda}^{-}(R \cup i)-v_{\lambda}^{-}(R)\right]\right. \text {, } \\
& \left.\lim _{\lambda \rightarrow \lambda^{*}} \sum_{R \subseteq N \backslash i} \gamma_{R}\left[v_{\lambda}^{+}(R \cup i)-v_{\lambda}^{+}(R)\right]\right] \\
& =\left[\sum_{R \subseteq N \backslash i} \gamma_{R}\left[v_{\lambda^{*}}^{-}(R \cup i)-v_{\lambda_{*}}^{-}(R)\right],\right. \\
& \left.\sum_{R \subseteq N \backslash i} \gamma_{R}\left[v_{\lambda^{*}}^{+}(R \cup i)-v_{\lambda^{*}}^{+}(R)\right]\right] \\
& =\sum_{R \subseteq N \backslash i} \gamma_{R}\left[\widetilde{v}_{\lambda^{*}}(R \cup i)-_{\mathrm{H}} \widetilde{v}_{\lambda_{*}}(R)\right] \\
& =\bar{\varphi}_{i}\left(\widetilde{v}_{\lambda^{*}}\right) \text {. }
\end{aligned}
$$

These complete the proof of Proposition 26.

Call players $i$ and $j$ symmetric in the game $(N, \widetilde{v})$ if $\widetilde{v}(S \cup$ $i)=\widetilde{v}(S \cup j)$ for every coalition $S \subseteq N \backslash\{i, j\}$. Call a player $i$ in a game $(N, \widetilde{v})$ a dummy (player) if $\widetilde{v}(S \cup i)-_{\mathrm{H}} \widetilde{v}(S)=\widetilde{v}(i)$ for all $S \subseteq N \backslash\{i\}$.

According to Theorem 24, it is easy to obtain the following conclusion.

Corollary 27. Given $\widetilde{v} \in F G_{0}(N)$, if $i$ and $j$ are symmetric in game $(N, \widetilde{v})$, then $\widetilde{\varphi}_{i}(\widetilde{v})=\widetilde{\varphi}_{j}(\widetilde{v})$. If $i$ is a dummy in game $(N, \widetilde{v})$, then $\widetilde{\varphi}_{i}(\widetilde{v})=\widetilde{v}(i)$. If $i$ is a null player in game $(N, \widetilde{v})$, then $\widetilde{\varphi}_{i}(\widetilde{v})=0$. 
Symmetric players have the same contribution to any coalition, and therefore it seems reasonable that they should obtain the same payoff according to the value. A dummy player only contributes his/her own worth to every coalition, and that is what he/she should be paid. A null player does not contribute anything to any coalition; in particular also $\widetilde{v}(i)=0$. So it seems reasonable that such a player obtains zero according to the value.

Corollary 28. Given $\widetilde{v} \in F G_{0}(N)$, for all $i \in N$,

$$
\widetilde{\varphi}_{i}(\widetilde{v}) \geq \widetilde{v}(i)
$$

Proof. Let $Q$ be a carrier of $\bar{v}$. If $i \in N$ and $i \notin Q$, then $\widetilde{v}(R \cup i)=$ $\widetilde{v}(R)$.

From $\widetilde{v}(R \cup i) \geq \widetilde{v}(R)+\widetilde{v}(i)$, we have $\widetilde{v}(i)=0$.

From Corollary 27, we have $\widetilde{\varphi}_{i}(\widetilde{v})=0$, so $\widetilde{\varphi}_{i}(\widetilde{v})=\widetilde{v}(i)$.

If $i \in N$ and $i \in Q$, since $\widetilde{v}(R \cup i) \geq \widetilde{v}(R)+\widetilde{v}(i)$, we have $\widetilde{v}(R \cup i)-_{\mathrm{H}} \widetilde{v}(R) \geq \widetilde{v}(i)$.

Hence, according to (44), we easily have $\widetilde{\varphi}_{i}(\widetilde{v}) \geq \widetilde{v}(i)$.

The proof is completed.

According to Theorem 24, it is easy to obtain the following conclusions, too.

Corollary 29. Define a function $\widetilde{\varphi}: F G_{0}(N) \rightarrow\left(\mathbb{R} \mathbb{R}^{+}\right)^{n}$ by

$$
\widetilde{\varphi}_{i}(\widetilde{v})(W)=\sum_{R \subseteq W \backslash i} \frac{r !(w-r-1) !}{w !}\left[\widetilde{v}(R \cup i)-_{\mathrm{H}} \widetilde{v}(R)\right],
$$$$
\text { if } i \in W \text {, }
$$

$$
\widetilde{\varphi}_{i}(\widetilde{v})(W)=0, \quad \text { if } i \notin W .
$$

Then the function $\widetilde{\varphi}$ is the unique fuzzy Shapley function on $F G_{0}(W)$.

Corollary 30. For any two $\widetilde{v}, \widetilde{w} \in F G_{0}(N)$, if $\widetilde{v}(S \cup i)-{ }_{\mathrm{H}} \widetilde{v}(S) \leq$ $\widetilde{w}(S \cup i)-_{\mathrm{H}} \widetilde{w}(S)$ for any $S \in P(N)$, then, for any $i \in N$,

$$
\widetilde{\varphi}_{i}(\widetilde{v}) \leq \widetilde{\varphi}_{i}(\widetilde{w})
$$

According to the above analysis, we can use another equivalent axiomatic system to define the fuzzy HukuharaShapley function on $F G_{0}(N)$.

Theorem 31. Given $\widetilde{v} \in F G_{0}(N)$, let $\tilde{\psi}: F G_{0}(N) \rightarrow\left(\mathbb{P} \mathbb{R}^{+}\right)^{n}$ be a value. Then $\tilde{\psi}$ satisfies the following three axioms, if and only if $\widetilde{\psi}(\widetilde{v})$ is the fuzzy Hukuhara-Shapley function $\widetilde{\varphi}(\widetilde{v})$.

Axiom $F_{1}$. If $\widetilde{v} \in F G_{0}(N)$, then

$$
\sum_{i \in N} \widetilde{\psi}_{i}(\widetilde{v})=\widetilde{v}(N)
$$

Axiom $F_{2}$. If players $i$ and $j$ are symmetricin the game $(N, \widetilde{v})$, then

$$
\widetilde{\psi}_{i}(\widetilde{v})=\widetilde{\psi}_{j}(\widetilde{v})
$$

Axiom $F_{3}$. For any two $\bar{v}, \widetilde{w} \in F G_{0}(N)$, if $\widetilde{v}(S \cup i)-_{\mathrm{H}} \widetilde{v}(S) \leq$ $\widetilde{w}(S \cup i)-_{\mathrm{H}} \widetilde{w}(S)$ for any $S \in P(N)$, then, for $\forall i \in N$,

$$
\widetilde{\psi}_{i}(\widetilde{v}) \leq \widetilde{\psi}_{i}(\widetilde{w}) \text {. }
$$

Proof. Obviously, $\widetilde{\varphi}(\widetilde{v})$ satisfies the three axioms. Conversely, suppose $\widetilde{\psi}(\widetilde{v})$ satisfies the three axioms.

(i) Let $\widetilde{z} \in F G_{0}(N)$ that is identically zero. In this fuzzy game, all players are symmetric, so Axiom $\mathrm{F}_{2}$ and Axiom $\mathrm{F}_{1}$ together imply $\widetilde{\psi}(\widetilde{z})=0$.

(ii) Let $i$ be a null player in $\widetilde{v} \in F G_{0}(N)$. Then the condition in Axiom $\mathrm{F}_{3}$ applies to $\widetilde{z}$ and $\widetilde{v}$ with all inequalities being equalities. So Axiom $\mathrm{F}_{3}$ yields $\widetilde{\psi}_{i}(\widetilde{v}) \geq \widetilde{\psi}_{i}(\widetilde{z})$ and $\widetilde{\psi}_{i}(\widetilde{z}) \geq \widetilde{\psi}_{i}(\widetilde{v})$. Hence, by $(\mathrm{i}), \widetilde{\psi}_{i}(\widetilde{v})=$ 0 .

(iii) Let $S \subseteq N \backslash \varnothing$ and $\widetilde{c}_{S} \in \mathbb{R}$. According to Lemma 20, we have

$$
\begin{gathered}
\widetilde{\psi}_{i}\left(\widetilde{c}_{S} u_{S}\right)=0 \quad \text { for every } i \in N \backslash S, \\
\widetilde{\psi}_{i}\left(\widetilde{c}_{S} u_{S}\right)=\frac{\widetilde{c}_{S}}{|S|} \quad \text { for every } i \in S .
\end{gathered}
$$

Hence, $\widetilde{\psi}\left(\widetilde{c}_{S} u_{S}\right)=\widetilde{c}_{S}|S|^{-1} e^{S}$, where $e^{S}=\left(s_{1}, s_{2}, \ldots, s_{n}\right)$ satisfying $s_{i}=1$ when $i \in S$ and $s_{i}=0$ when $i \in N \backslash S$.

(iv) According to Lemma 23 we have

$$
\widetilde{v}=\sum_{S \in P(N) \backslash \varnothing} \tilde{c}_{S} u_{S}
$$

The proof of $\widetilde{\psi}(\widetilde{v})=\widetilde{\varphi}(\widetilde{v})$ is completed by induction on the number $\alpha(\widetilde{v})$ of terms in $\widetilde{v}=\sum_{S \in P(N) \backslash \varnothing} \widetilde{c}_{S} u_{S}$ with $\widetilde{c}_{S} \neq 0$.

From (i), $\widetilde{\psi}(\widetilde{v})=\widetilde{\varphi}(\widetilde{v})=0$ if $\alpha(\widetilde{v})=0$ and, from (iii), $\widetilde{\psi}(\widetilde{v})=\widetilde{\varphi}(\widetilde{v})$ if $\alpha(\widetilde{v})=1$ because of $\widetilde{\phi}\left(\widetilde{c}_{S} u_{S}\right)=\widetilde{c}_{S}|S|^{-1} e^{T}$. Suppose $\widetilde{\psi}(\widetilde{w})=\widetilde{\varphi}(\widetilde{w})$ for all $\widetilde{w} \in F G_{0}(N)$ with $\alpha(\widetilde{w})<k$, where $k \geq 2$. Let $\widetilde{v} \in F G_{0}(N)$ with $\alpha(\widetilde{v})=k$. Then there exist coalitions $S_{1}, S_{2}, \ldots, S_{k}$ and fuzzy numbers $\widetilde{c}_{1}, \widetilde{c}_{2}, \ldots, \widetilde{c}_{k}$ unequal to zero, such that $\tilde{v}=\sum_{r=1}^{k} \widetilde{c}_{r} u_{S_{r}}$. Let $D=\bigcap_{r=1}^{k} S_{r}$.

For $i \in N \backslash D$, define $\widetilde{w}^{i}=\sum_{r: i \in S_{r}} \widetilde{r}_{r} \widetilde{\mu}_{S_{r}}$. Because $\alpha\left(\widetilde{w}^{i}\right)<k$, the induction hypothesis implies

$$
\widetilde{\psi}_{i}\left(\widetilde{w}^{i}\right)=\widetilde{\varphi}_{i}\left(\widetilde{w}^{i}\right)
$$

Further, for every $T \in P(N)$,

$$
\begin{aligned}
& \widetilde{v}(T \cup i)-_{\mathrm{H}} \widetilde{v}(T) \\
& =\sum_{r=1}^{k} \widetilde{c}_{r} u_{S_{r}}(T \cup i)-_{\mathrm{H}} \sum_{r=1}^{k} \widetilde{c}_{r} u_{S_{r}}(T) \\
& =\sum_{r: i \in S_{r}} \widetilde{c}_{r} u_{S_{r}}(T \cup i)-_{\mathrm{H}} \sum_{r: i \in S_{r}} \widetilde{c}_{r} u_{S_{r}}(T) \\
& =\widetilde{w}^{i}(T \cup i)-_{\mathrm{H}} \widetilde{w}^{i}(T) .
\end{aligned}
$$

So, by Axiom $\mathrm{F}_{2}$, it follows that $\widetilde{\psi}_{i}(\widetilde{v})=\widetilde{\psi}_{i}\left(\widetilde{w}^{i}\right)=\widetilde{\phi}_{i}\left(\widetilde{w}^{i}\right)=$ $\widetilde{\phi}_{i}(\widetilde{v})$. So, for all $i \in N \backslash D$, we have

$$
\widetilde{\psi}_{i}(\widetilde{v})=\widetilde{\varphi}_{i}(\widetilde{v}) .
$$


TABLE 1: Interval Hukuhara-Shapley values for different $\lambda$-level of decision-makers.

\begin{tabular}{lcccc}
\hline & $\lambda=0.8$ & $\lambda=0.6$ & $\lambda=0.4$ & $\lambda=0.2$ \\
\hline $\bar{\varphi}_{1}\left(\widetilde{v}_{\lambda}\right)$ & {$[19.8,20.3]$} & {$[19.5,20.6]$} & {$[19.3,20.9]$} & {$[19.1,21.2]$} \\
$\bar{\varphi}_{2}\left(\widetilde{v}_{\lambda}\right)$ & {$[17.0,17.9]$} & {$[16.4,18.3]$} & {$[15.9,18.7]$} & {$[15.4,19.1]$} \\
$\bar{\varphi}_{3}\left(\widetilde{v}_{\lambda}\right)$ & {$[12.3,12.8]$} & {$[12.0,13.1]$} & {$[11.8,13.4]$} & {$[11.6,13.7]$} \\
\hline
\end{tabular}

Further, according to Axiom $\mathrm{F}_{1}$, combining with (61), we have

$$
\sum_{i \in D} \widetilde{\psi}_{i}(\widetilde{v})=\sum_{i \in D} \widetilde{\varphi}_{i}(\widetilde{v})
$$

Let $i, j \in D$; then, for every $T \subseteq N \backslash i, j$,

$$
\begin{aligned}
\widetilde{v}(T \cup i) & =\sum_{r=1}^{k} \widetilde{c}_{r} u_{S_{r}}(T \cup i) \\
& =\sum_{r=1}^{k} \widetilde{c}_{r} u_{S_{r}}(T \cup j)=\widetilde{v}(T \cup j),
\end{aligned}
$$

so $i$ and $j$ are symmetric. Hence, by Axiom $\mathrm{F}_{2}$, we have

$$
\widetilde{\psi}_{i}(\widetilde{v})=\widetilde{\psi}_{j}(\widetilde{v}), \quad \widetilde{\varphi}_{i}(\widetilde{v})=\tilde{\varphi}_{j}(\widetilde{v})
$$

From (61), (62), and (64), we have $\widetilde{\psi}(\widetilde{v})=\widetilde{\varphi}(\widetilde{v})$.

The proof is completed.

Example 32. Consider three economic companies, named 1,2 , and $3(i=1,2,3)$. They possess different resources. Now they want to do a joint project by means of pooling their resources. It is natural for these three decision-makers to try to evaluate the revenue of the joint project in the early period of the project in order to decide whether the project can be realized or not. However, the average profit of the joint project is dependent on a number of companies. And the average profit of the joint project is an approximate evaluation, which is represented by fuzzy numbers as follows:

$$
\begin{gathered}
\widetilde{v}(i)(x)=\left\{\begin{array}{ll}
x-4, & x \in[4,5] \\
6-x, & x \in[5,6] \\
0, & \text { otherwise, }
\end{array} \quad i=1,2,3 .\right. \\
\widetilde{v}(1,2)(x)= \begin{cases}\frac{x}{5}-6, & x \in[30,35] \\
\frac{38}{3}-\frac{x}{3}, & x \in[35,38] \\
0, & \text { otherwise, }\end{cases}
\end{gathered}
$$

$$
\begin{gathered}
\widetilde{v}(1,3)(x)= \begin{cases}\frac{x}{2}-\frac{23}{2}, & x \in[23,25] \\
\frac{27}{2}-\frac{x}{2}, & x \in[25,27] \\
0, & \text { otherwise },\end{cases} \\
\widetilde{v}(2,3)(x)= \begin{cases}\frac{x}{5}-3, & x \in[15,20] \\
\frac{23}{3}-\frac{x}{3}, & x \in[20,23] \\
0, & \text { otherwise, }\end{cases} \\
\widetilde{v}(1,2,3)(x)= \begin{cases}\frac{x}{5}-9, & x \in[45,50] \\
11-\frac{x}{5}, & x \in[50,55] \\
0, & \text { otherwise },\end{cases} \\
\widetilde{v}(\varnothing)=0 .
\end{gathered}
$$

According to (44), we can calculate fuzzy HukuharaShapley function of each decision-maker as follows:

$$
\begin{aligned}
& \widetilde{\varphi}_{1}(\widetilde{v})(x)= \begin{cases}\frac{6}{7} x-\frac{113}{7}, & x \in\left[\frac{113}{6}, 20\right] \\
\frac{43}{3}-\frac{2}{3} x, & x \in\left[20, \frac{43}{2}\right] \\
0, & \text { otherwise, }\end{cases} \\
& \widetilde{\varphi}_{2}(\widetilde{v})(x)= \begin{cases}\frac{3}{8} x-\frac{89}{16}, & x \in\left[\frac{89}{6}, \frac{35}{2}\right] \\
\frac{39}{4}-\frac{1}{2} x, & x \in\left[\frac{35}{2}, \frac{39}{2}\right] \\
0, & \text { otherwise, }\end{cases} \\
& \widetilde{\varphi}_{3}(\widetilde{v})(x)= \begin{cases}\frac{6}{7} x-\frac{68}{7}, & x \in\left[\frac{34}{3}, \frac{25}{2}\right] \\
\frac{28}{3}-\frac{2}{3} x, & x \in\left[\frac{25}{2}, \frac{24}{2}\right] \\
0, & \text { otherwise. }\end{cases}
\end{aligned}
$$

By judging the allocations of fuzzy Hukuhara-Shapley function of each decision-maker, decision-makers can conclude whether the joint project can be realized or not. According to fuzzy Hukuhara-Shapley function, it is very convenient for us to obtain their interval Hukuhara-Shapley values of different $\lambda$-level as shown in Table 1 . 


\section{Generalized Fuzzy Shapley Function for Generalized Fuzzy Games}

4.1. Cooperative Games with Fuzzy Coalition. For a finite set of players $N=\{1,2, \ldots, n\}$, we call $x=\left(x_{1}, x_{2}, \ldots, x_{n}\right)$, $x_{i} \in[0,1]$, the fuzzy coalition variable and call $x^{\#}=\{y \mid$ $y_{i}=x_{i}$ or $y_{i}=0$ for each $\left.i \in N\right\}$ the set of fuzzy coalition variables created by $x$. The level variable set of $x$ is denoted by $L x=\left\{x_{1}, x_{2}, \ldots, x_{n}\right\}$. For any $A \subseteq N$, we denote $L A=\left\{x_{i} \mid\right.$ $i \in A\} . s=\left(s_{1}, s_{2}, \ldots, s_{n}\right)$ is called a fuzzy coalition; here, $s_{i}$ is a constant which denotes the participation level of player $i$. Similar to $x^{\#}$, we denote by $s^{\#}$ the class of fuzzy coalitions created by $s$, which is defined by $s^{\#}=\left\{t \mid t_{i}=s_{i}\right.$ or $t_{i}=$ 0 for each $i \in N\}$. The set of fuzzy coalitions is denoted by $L(N)$; the empty coalition in a fuzzy setting is denoted by $e^{\varnothing}=(0,0, \ldots, 0) \cdot e^{S}=\left(s_{1}, s_{2}, \ldots, s_{n}\right)$ satisfying $s_{i}=1$ when $i \in S$ and $s_{i}=0$ when $i \in N \backslash S$, with $S \in P(N)$, denotes a crisp coalition. It corresponds to the situation where the players within $S$ fully cooperate; that is, they have participation level 1 , and the players outside $S$ are not involved in at all; that is, they have participation level $0 . e^{N}=(1,1, \ldots, 1)$ is called the grand coalition. We write $e^{i}$ instead of $e^{\{i\}}$. Let $T \subseteq N$; the fuzzy coalition variable $x_{T}$ corresponding to $T$ is denoted by $x_{T}=\sum_{k \in T} x_{k} e^{k}$. When $x=s$, the fuzzy coalition $s_{T}$ corresponding to $T$ is denoted by $s_{T}=\sum_{k \in T} x_{k} e^{k}$.

Cooperative games with fuzzy coalitions are represented by a pair $(N, v)$, where function $v: L(N) \rightarrow \mathbb{R}^{+}$such that $v(\varnothing)=0$. If there is no fear of confusion, it is denoted by $v$ as well. The class of cooperative games with fuzzy coalitions and player set $N$ is denoted by $L G(N)$.

In what follows, $s \vee t$ and $s \wedge t$ are those elements of $L(N)$ with the $i$ th coordinate equal to $\max \left(s_{i}, t_{i}\right)$ and $\min \left(s_{i}, t_{i}\right)$, respectively. The set operations $\vee$ and $\wedge$ play the same role for the fuzzy coalitions as the union and intersection for crisp coalitions. For any $s, t \in L(N), s \wedge t=\varnothing$, if $v(s \vee t) \geq v(s)+v(t)$, then $v$ is called superadditive game.

Definition 33. Given $v \in L G(N), s \in L(N)$. Based on $s$, the participation level $s_{i}$ of player $i$ is called a dummy participation level if, for any $t \in\left(s-\sum_{k \in N \backslash i} s_{k} e^{k}\right)^{\#}, v\left(s_{i} e^{i} \vee t\right)=$ $v\left(s_{i} e^{i}\right)+v(t)$. And the participation level $s_{i}$ of player $i$ is called a null participation level if, for any $t \in\left(s-\sum_{k \in N \backslash i} s_{k} e^{k}\right)^{\#}$, $v\left(s_{i} e^{i} \vee t\right)=v(t)$.

Definition 34. Given $v \in L G_{0}(N), s \in L(N)$; based on $s$, a fuzzy coalition $\bar{s} \in s^{\#}$ is called a carrier of $s$ in $v$ if, for any $t \in s^{\#}, v(t)=v(t \wedge \bar{s})$.

The set of carriers of $s$ in $v$ is denoted by $\operatorname{SC}(v, s)$.

Let $\pi$ be a permutation of $N$. For any $s \in L(N)$ and any $v \in L G_{0}(N)$, the permutation of fuzzy coalition $s$ is defined by $\pi s=s \cdot \pi^{-1}$ and $\pi v(s)=v\left(\pi^{-1} s\right)$. Then, $\pi v: s \mapsto \pi v(s)$ from $L(N)$ to $\mathbb{R}^{+}$is still a fuzzy game in $L G_{0}(N)$.

A crisp coalition $S \in 2^{N}$ corresponds in a canonical way with fuzzy coalition $e_{S}$, where $e^{S} \in[0,1]^{N}$ is the vector $e^{S}=$ $\left\{e^{S}(1), e^{S}(2), \ldots, e^{S}(n)\right\}$, where the $i$ th coordinate $e^{S}(i)=1$ if and only if $i \in S$; otherwise, $e^{S}(i)=0$. From the viewpoint of geometry meaning, the set $2^{N}$ of all admissible coalitions in
$N$ is the set of all vertexes of $[0,1]^{N}$. Therefore, the set $2^{N}$ of all admissible coalitions can be equivalently denoted by $\{0,1\}^{N}$ : $S \in 2^{N} \rightarrow e_{S} \in\{0,1\}^{N}$; that is, the correspondence between $2^{N}$ and $\{0,1\}^{N}$ is one to one. Through this identification of coalitions with their characteristic vectors, a cooperative game is a function $v:\{0,1\}^{N} \rightarrow \mathbb{R}^{+}$with $v(O)=0$, where $\mathrm{O}=(0,0, \ldots, 0)$ corresponding to the empty coalition.

There exist different methods to extend $n$-person cooperative games to $n$-person cooperative games with fuzzy coalitions, such as proportional values method [5] and Choquet integral method $[6,26]$. However, Li and Zhang [9] pointed out that which one is more natural may be insignificant if the specific game situation is not considered. Each fuzzy game may only be suitable for a certain case. When a fuzzy game established in one case is applied to another situation, the invalidity of it may be inevitable. Inspired by their work, in the following, we propose a generalized fuzzy game, game with fuzzy coalitions and fuzzy characteristic function, and a simplified expression of the fuzzy Shapley function is given for this generalized fuzzy game. It is also proved that the simplified expression is regarded as the generalization of fuzzy Shapley function defined in some particular fuzzy games, such as fuzzy games with proportional value and Choquet integral form.

4.2. Generalized Fuzzy Shapley Function for Generalized Fuzzy Games. In this section, we incorporate fuzzy coalition and fuzzy characteristic function into the game, which is called a generalized fuzzy game or fuzzy game.

A generalized fuzzy game is represented by a pair $(N, \widetilde{w})$, where the fuzzy characteristic function $\widetilde{w}: L(N) \rightarrow \mathbb{R} \mathbb{R}^{+}$ with $\widetilde{w}(\varnothing)=0$.

Obviously, a fuzzy game degenerates to be a game with fuzzy coalitions when the range of the mapping $\widetilde{w}$ is $\mathbb{R}^{+}$and to be a game with a fuzzy characteristic function when the definitional domain of the mapping $\widetilde{w}$ is $P(N)$. It degenerates to be a crisp game when the definitional domain and range of the mapping $\widetilde{w}$ are $P(N)$ and $\mathbb{R}^{+}$, respectively. Thus, our discussion and game models can be applicable to games involving both crisp and fuzzy games. The set of games with fuzzy coalition and fuzzy characteristic function is denoted by $\operatorname{LFG}(N)$.

Definition 35. Given $\widetilde{w} \in L F G(N), s \in L(N)$. A function $\widetilde{\varphi}\left(\widetilde{w}^{s}\right): \operatorname{LFG}(N) \rightarrow\left(\mathbb{F R}^{+}\right)^{n}$ is called generalized fuzzy Shapley function based on $s$ if it satisfies the following three axioms.

Axiom $S_{1}$. If $\widetilde{w} \in \operatorname{LFG}(N), \bar{s} \in S C(\widetilde{w}, s)$, then

$$
\sum_{i \in N} \widetilde{\varphi}_{i}^{s}(\widetilde{w})=\widetilde{w}(\bar{s})
$$

Axiom $S_{2}$. If $\widetilde{w} \in \operatorname{LFG}(N)$ and $\pi$ is a permutation of $s$, then, for any $i \in N$,

$$
\widetilde{\varphi}_{\pi i}^{s}(\pi \widetilde{w})=\widetilde{\varphi}_{i}^{s}(\widetilde{w})
$$


Axiom $S_{3}$. For any $\widetilde{w}_{1}, \widetilde{w}_{2} \in L F G(N)$, if a game is defined by $\left(\widetilde{w}_{1}+\widetilde{w}_{2}\right)(t)=\widetilde{w}_{1}(t)+\widetilde{w}_{2}(t)$ for any $t \in s^{\#}$, then

$$
\widetilde{\varphi}_{i}^{s}\left(\widetilde{w}_{1}+\widetilde{w}_{2}\right)=\widetilde{\varphi}_{i}^{s}\left(\widetilde{w}_{1}\right)+\widetilde{\varphi}_{i}^{s}\left(\widetilde{w}_{2}\right) \quad \forall i \in N .
$$

Theorem 36. Given $\widetilde{w} \in \operatorname{LFG}(N), x=\left(x_{1}, x_{2}, \ldots, x_{n}\right)$ is fuzzy coalition variable, $s=\left(s_{1}, s_{2}, \ldots, s_{n}\right) \in L(N)$. Then

$$
\begin{array}{r}
\left.\widetilde{\varphi}_{x_{i}}(\widetilde{w})\right|_{x=s} \\
=\sum_{R \subseteq N \backslash i} \frac{r !(n-r-1) !}{n !}\left[\widetilde{w}\left(\sum_{j \in R \cup i} s_{j} e^{j}\right){ }_{H} \widetilde{w}\left(\sum_{j \in R} s_{j} e^{j}\right)\right], \\
i=1,2, \ldots, n
\end{array}
$$

is a generalized fuzzy Shapley function of player $i$ with participation level $s_{i}$ based on $s$.

Proof. It is similar to the proof of fuzzy Hukuhara-Shapley function in Theorem 24; therefore, here it is omitted.

By means of Choquet integral, Yu and Zhang [18] proposed a class of fuzzy games with indeterminate integral form and the fuzzy Shapley function was developed.

Definition 37. Given $s \in L(N)$, let $Q(s)=\left\{s_{i} \mid s_{i}>0, i \in N\right\}$ and let $q(s)$ be the cardinality of $Q(s)$; that is, $q(s)=|Q(s)|$. The elements of $Q(s)$ are written in the increasing order as $h_{1}<\cdots<h_{q(s)}$. Then a game $\widetilde{w} \in \operatorname{LFG}(N)$ is said to be a fuzzy game with "indeterminate integral form" if and only if the following holds:

$$
\widetilde{w}(s)=\sum_{l=1}^{q(s)} \widetilde{w}\left([s]_{h_{l}}\right)\left(h_{l}-h_{l-1}\right),
$$

for any $s \in L(N)$, where $h_{0}=0$.

The set of games with indeterminate integral form in $L F G(N)$ is denoted by $L F G_{C}(N)$.

For $\widetilde{w} \in L F G_{C}(N), Y u$ and Zhang [18] obtained the following conclusion.

Theorem 38. The function $\tilde{f}: \operatorname{LFG}_{C}(N) \rightarrow\left(\mathbb{F} \mathbb{R}_{+}^{n}\right)^{L(N)}$ defined by

$$
\widetilde{f}_{i}(\widetilde{w})(s)=\sum_{l=1}^{q(s)} \widetilde{\varphi}_{i}(\widetilde{w})\left([s]_{h_{l}}\right)\left(h_{l}-h_{l-1}\right)
$$

is a fuzzy Shapley function on $\operatorname{LFG}_{C}(N)$, where $\tilde{\varphi}$ is the function given by (44).

Besides Choquet integral method, we can apply the proportional values method proposed by Butnariu [5] to obtain a special game with fuzzy coalition and fuzzy characteristic function.

Let $s$ be a fuzzy coalition and $r \in[0,1]$. $r$-section of $s$ is denoted by $s^{r}=\left\{i \mid i \in N, s_{i}=r\right\}$. The r-section of $s$ is the set of players, in which each player's participation level is $r$. Based on the definition of $r$-section, we define a fuzzy game with proportional values.
Definition 39. The game $\widetilde{w} \in \operatorname{LFG}(N)$ is said to be a fuzzy game with proportional values if and only if for any $s$ in $L(N)$ the following holds:

$$
\widetilde{w}(s)=\sum_{r \in[0,1]} \widetilde{w}\left(s^{r}\right) \cdot r .
$$

The set of the fuzzy games with proportional values in $L F G(N)$ will be denoted by $L F G_{P}(N)$. For the sake of simplicity, a fuzzy game with proportional values form is called a fuzzy game if there is no fear of confusion. There is a one-to-one correspondence between a game with a fuzzy characteristic function and a fuzzy game. We call the game with a fuzzy characteristic function corresponding to a fuzzy game the associated game with a fuzzy characteristic function, and we call the fuzzy game corresponding to a game with a fuzzy characteristic function the associated fuzzy game.

Theorem 40. A function $\tilde{f}: L F G_{P}(N) \rightarrow\left(\mathbb{P R}_{+}^{n}\right)^{L(N)}$ defined by

$$
\widetilde{g}_{i}(\widetilde{w})(s)=\widetilde{\varphi}_{i}(\widetilde{w})\left(s^{r}\right) \cdot s_{i}
$$

is a fuzzy Shapley function of player $i$ with participation level $s_{i}$ on $\operatorname{LFG}_{C}(N)$, where $\tilde{\varphi}$ is the function given by (44).

Proof. We only need to prove that the function defined by (74) satisfies Axioms $S_{1}-S_{3}$ in Definition 35. Similar to the proof of Theorem 24, it is easy to obtain the result. Therefore, here it is omitted.

In the following, we make a comparison among the above fuzzy Shapley functions.

For any $S \subseteq N$, we denote by $N(S)$ the set of $n$-players with the players of $N \backslash S$ being null players of $v$. Then, for any $T \subseteq N(S)$ and $\widetilde{v} \in F G(N)$, we have $\widetilde{v}(T)=\widetilde{v}(T \cap S)$.

Lemma 41. Let $\widetilde{v} \in F G(N) ; \widetilde{\varphi}_{i}(\widetilde{v})$ is a fuzzy HukuharaShapley function in Theorem 24. Then, for any $S \in P(N)$,

$$
\widetilde{\varphi}_{i}(\widetilde{v})(S)=\widetilde{\varphi}_{i}(\widetilde{v})(N(S)) .
$$

Proof. According to the definition of $\widetilde{\varphi}_{i}(\widetilde{v})$, the proof of Lemma 41 is similar to that of Lemma 1 in Li and Zhang [9] and therefore here it is omitted.

Theorem 42. Let $\widetilde{w} \in L F G_{C}(N), s=\left(s_{1}, s_{2}, \ldots, s_{n}\right) \in L(N)$. Then, for any $i \in N$,

$$
\left.\widetilde{\varphi}_{x_{i}}(\widetilde{w})\right|_{x=s}=\widetilde{f}_{i}(\widetilde{w})(s) .
$$

Proof. Let $Q(s)=\left\{s_{i} \mid s_{i}>0, i \in N\right\}, q=|Q(s)|$. The elements of $Q(s)$ are written in the increasing order as $h_{1}<\cdots<h_{q}$. Suppose $s_{i}=h_{k}$; then

$$
\begin{aligned}
\widetilde{f}_{i}(\widetilde{w})(s)= & \sum_{l=1}^{q(s)} \widetilde{\varphi}_{i}(\widetilde{w})\left([s]_{h_{1}}\right)\left(h_{l}-h_{l-1}\right) \\
= & \widetilde{\varphi}_{i}(\widetilde{w})\left([s]_{h_{1}}\right) h_{1}+\widetilde{\varphi}_{i}(\widetilde{w})\left([s]_{h_{2}}\right)\left(h_{2}-h_{1}\right) \\
& +\cdots+\widetilde{\varphi}_{i}(\widetilde{w})\left([s]_{h_{q}}\right)\left(h_{q}-h_{q-1}\right) .
\end{aligned}
$$


By Corollary 29, it is obvious that $\widetilde{\varphi}_{i}(\widetilde{w})\left([s]_{h_{j}}\right)=0$ for any $h_{j}>h_{k}$. Then

$$
\begin{aligned}
\widetilde{f}_{i}(\widetilde{w})(s)= & \widetilde{\varphi}_{i}(\widetilde{w})\left([s]_{h_{1}}\right) h_{1}+\widetilde{\varphi}_{i}(\widetilde{w})\left([s]_{h_{2}}\right) \\
& \times\left(h_{2}-h_{1}\right)+\cdots+\widetilde{\varphi}_{i}(\widetilde{w})\left([s]_{h_{k}}\right) \\
& \times\left(h_{k}-h_{k-1}\right) .
\end{aligned}
$$

According to Lemma 41, the remaining part of the proof of Theorem 42 is similar to that of Theorem 4.3 in Li and Zhang [9]; therefore, here it is omitted.

Theorem 43. Let $\widetilde{w} \in L F G_{P}(N), s=\left(s_{1}, s_{2}, \ldots, s_{3}\right) \in L(N)$. Then, for any $i \in N$,

$$
\left.\widetilde{\varphi}_{x_{i}}(\widetilde{w})\right|_{x=s}=\widetilde{g}_{i}(\widetilde{w})(s) .
$$

Proof. From Lemma 41, the proof of Theorem 43 is similar to that of Theorem 4.1 in Li and Zhang [9]; therefore, here it is omitted.

Remark 44. From the above analysis, itis easily seen that the simplified expression of the generalized fuzzy Shapley function given by (70) can be regarded as the generalization of fuzzy Shapley functions defined in some particular fuzzy games with fuzzy coalition. The simplified expression of the fuzzy Shapley function is equivalent to $\mathrm{Yu}$ and Zhang's definition [18] when fuzzy characteristic function is a fuzzy game with Choquet integral forms and is equivalent to that when fuzzy characteristic function is a fuzzy game with proportional values.

Remark 45. The generalized fuzzy Shapley function given by (70) can be applied to crisp games, games with fuzzy coalitions, and games with fuzzy characteristic functions by restricting the domain. Equation (70) coincides with (44) or (52) when restricted to a crisp coalition; (70) is equal to (17) when restricted to a real-valued characteristic function and a crisp coalition. Briefly, the generalized fuzzy Shapley function defined by (70) is a general Shapley function, which serves as the connection between games with fuzzy coalitions and games with fuzzy characteristic functions. The most important aspect is that we do not need to transform the Shapley function when dealing with different kinds of cooperative games.

\section{Conclusion}

Shapley value is a well-known solution concept in cooperative game theory. In crisp game, it has been applied widely in many cases. In fuzzy game, Shapley functions of game with fuzzy coalition or with fuzzy characteristic function, as an important solution concept, have been paid more attention. Games, subject to fuzzy coalitions as well as those pertaining to fuzzy characteristic function, are separately investigated in the literature. In this paper, based on the Hukuhara difference, a fuzzy Hukuhara-Shapley function on the class of games with fuzzy characteristic function is investigated.
And some interesting properties are shown. Further, a new fuzzy game model that admits both fuzzy coalitions and fuzzy characteristic functions is proposed. A simplified expression of the generalized fuzzy Shapley function for the generalized fuzzy games is given. It is shown that the simplified expression of the generalized fuzzy Shapley function is equivalent to that of fuzzy games with indeterminate integral form in the study by Yu and Zhang [18]. When the domain of the characteristic function or coalition is restricted, the generalized fuzzy Shapley function can be applicable to crisp games, games with fuzzy coalitions, and games with fuzzy characteristic functions. Thus, the simplified expression of the generalized fuzzy Shapley function for this kind of fuzzy games can be widely applied in many cases to address more realistic situations.

\section{Conflict of Interests}

The authors declare that they have no conflict of interests regarding the publication of this paper.

\section{Acknowledgments}

This work was partly supported by the Funds for Creative Research Groups of China (no. 71221061), the Projects of Major International Cooperation NSFC (no. 71210003), the National Natural Science Foundation of China (nos. 71271217 and 71201089), and the Program for New Century Excellent Talents in University of China (no. NCET-12-0541).

\section{References}

[1] L. S. Shapley, "A value for $n$-person games," in Contributions to the Theory of Games, vol. 2 of Annals of Mathematics Studies, No. 28, pp. 307-317, Princeton University Press, Princeton, NJ, USA, 1953.

[2] J. P. Aubin, "Coeur et valeur des jeux flous à paiements latéraux," Comptes Rendus Hebdomadaires des Séances de 1'Académie des Sciences A, vol. 279, pp. 891-894, 1974.

[3] J. Aubin, Mathematical Methods of Game and Economic Theory, North-Holland Publishing, Amsterdam, The Netherlands, 1979.

[4] A. Billot, "Fuzzy convexity and peripheral core of an exchange economy represented as a fuzzy game," in Multiperson Decision Making Models Using Fuzzy Sets and Possibility Theory, J. Kacprzyk and M. Fedrizzi, Eds., vol. 18, pp. 311-335, Kluwer Academic Publishers, Dordrecht, The Netherlands, 1990.

[5] D. Butnariu, "Stability and Shapley value for an $n$-persons fuzzy game," Fuzzy Sets and Systems, vol. 4, no. 1, pp. 63-72, 1980.

[6] M. Tsurumi, T. Tanino, and M. Inuiguchi, "A Shapley function on a class of cooperative fuzzy games," European Journal of Operational Research, vol. 129, no. 3, pp. 596-618, 2001.

[7] R. Branzei, D. Dimitrov, and S. Tijs, Models in Cooperative Game Theory: Crisp, Fuzzy and Multichoice Games, vol. 556 of Lecture Notes in Economics and Mathematical Systems, Springer, New York, NY, USA, 2005.

[8] D. Butnariu and T. Kroupa, "Shapley mappings and the cumulative value for $n$-person games with fuzzy coalitions," European Journal of Operational Research, vol. 186, no. 1, pp. 288-299, 2008. 
[9] S. Li and Q. Zhang, "A simplified expression of the Shapley function for fuzzy game," European Journal of Operational Research, vol. 196, no. 1, pp. 234-245, 2009.

[10] G. Owen, "On the core of linear production games," Mathematical Programming, vol. 9, no. 3, pp. 358-370, 1975.

[11] I. Nishizaki and M. Sakawa, "Fuzzy cooperative games arising from linear production programming problems with fuzzy parameters," Fuzzy Sets and Systems, vol. 114, no. 1, pp. 11-21, 2000.

[12] M. Sakawa, Fuzzy Sets and Interactive Multiobjective Optimization, Plenum Press, New York, NY, USA, 1993.

[13] M. Sakawa and H. Yano, "An interactive fuzzy satisficing method for generalized multiobjective linear programming problems with fuzzy parameters," Fuzzy Sets and Systems, vol. 35, no. 2, pp. 125-142, 1990.

[14] M. Mares, "Fuzzy coalition structures," Fuzzy Sets and Systems, vol. 114, no. 1, pp. 23-33, 2000.

[15] M. Mares, Fuzzy Cooperative Games: Cooperation with Vague Expectations, vol. 72 of Studies in Fuzziness and Soft Computing, Physica, New York, NY, USA, 2001.

[16] M. Mares and M. Vlach, "Linear coalitional games and their fuzzy extensions," International Journal of Uncertainty, Fuzziness and Knowledge-Based Systems, vol. 9, no. 3, pp. 341-354, 2001.

[17] S. Borkotokey, "Cooperative games with fuzzy coalitions and fuzzy characteristic functions," Fuzzy Sets and Systems, vol. 159, no. 2, pp. 138-151, 2008.

[18] X. Yu and Q. Zhang, "An extension of cooperative fuzzy games," Fuzzy Sets and Systems, vol. 161, no. 11, pp. 1614-1634, 2010.

[19] G. Alefeld and J. Herzberger, Introduction to Interval Computations, Academic Press, New York, NY, USA, 1983.

[20] H. T. Banks and M. Q. Jacobs, "A differential calculus for multifunctions," Journal of Mathematical Analysis and Applications, vol. 29, pp. 246-272, 1970.

[21] D. Dubois, E. Kerre, R. Mesiar, and H. Prade, "Fuzzy interval analysis," in Fundamentals of Fuzzy Sets, D. Dubois and H. Prade, Eds., vol. 7 of The Handbooks of Fuzzy Sets Series, pp. 483-581, Kluwer Academic Publishers, Boston, Mass, USA, 2000.

[22] L. Stefanini, "A generalization of Hukuhara difference and division for interval and fuzzy arithmetic," Fuzzy Sets and Systems, vol. 161, no. 11, pp. 1564-1584, 2010.

[23] L. A. Zadeh, "Fuzzy sets," Information and Computation, vol. 8, pp. 338-353, 1965.

[24] L. A. Zadeh, "The concept of a linguistic variable and its application to approximate reasoning I," Information Sciences, vol. 8, pp. 199-249, 1975.

[25] G. J. Klir and B. Yuan, Fuzzy Sets and Fuzzy Logic Theory and Applications, Prentice Hall, New Jersey, NJ, USA, 1995.

[26] C. Q. Tan, Z. Z. Jiang, and X. H. Chen, "Choquet extension of cooperative games," Asia-Pacific Journal of Operational Research, vol. 30, no. 4, Article ID 1350005, 20 pages, 2013. 


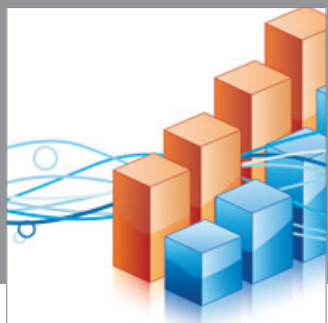

Advances in

Operations Research

mansans

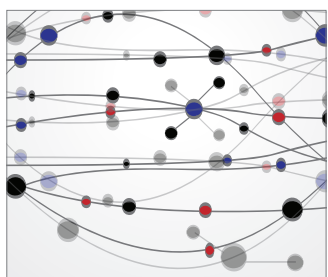

The Scientific World Journal
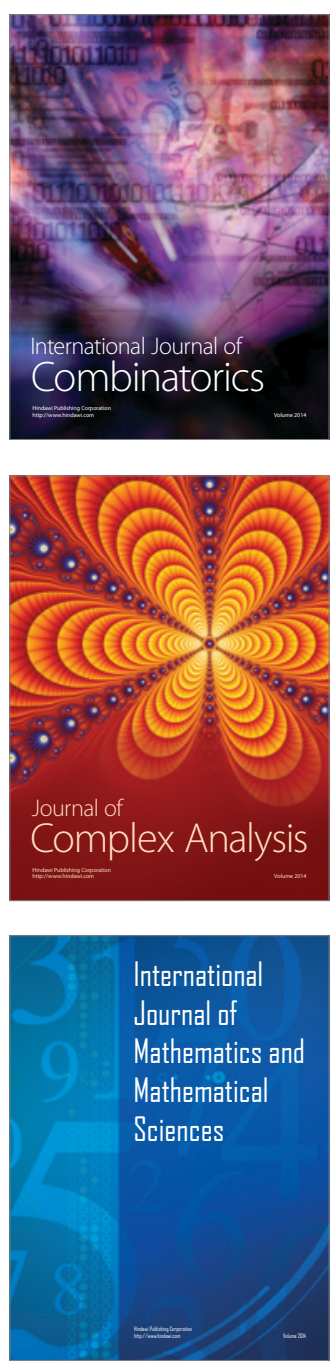
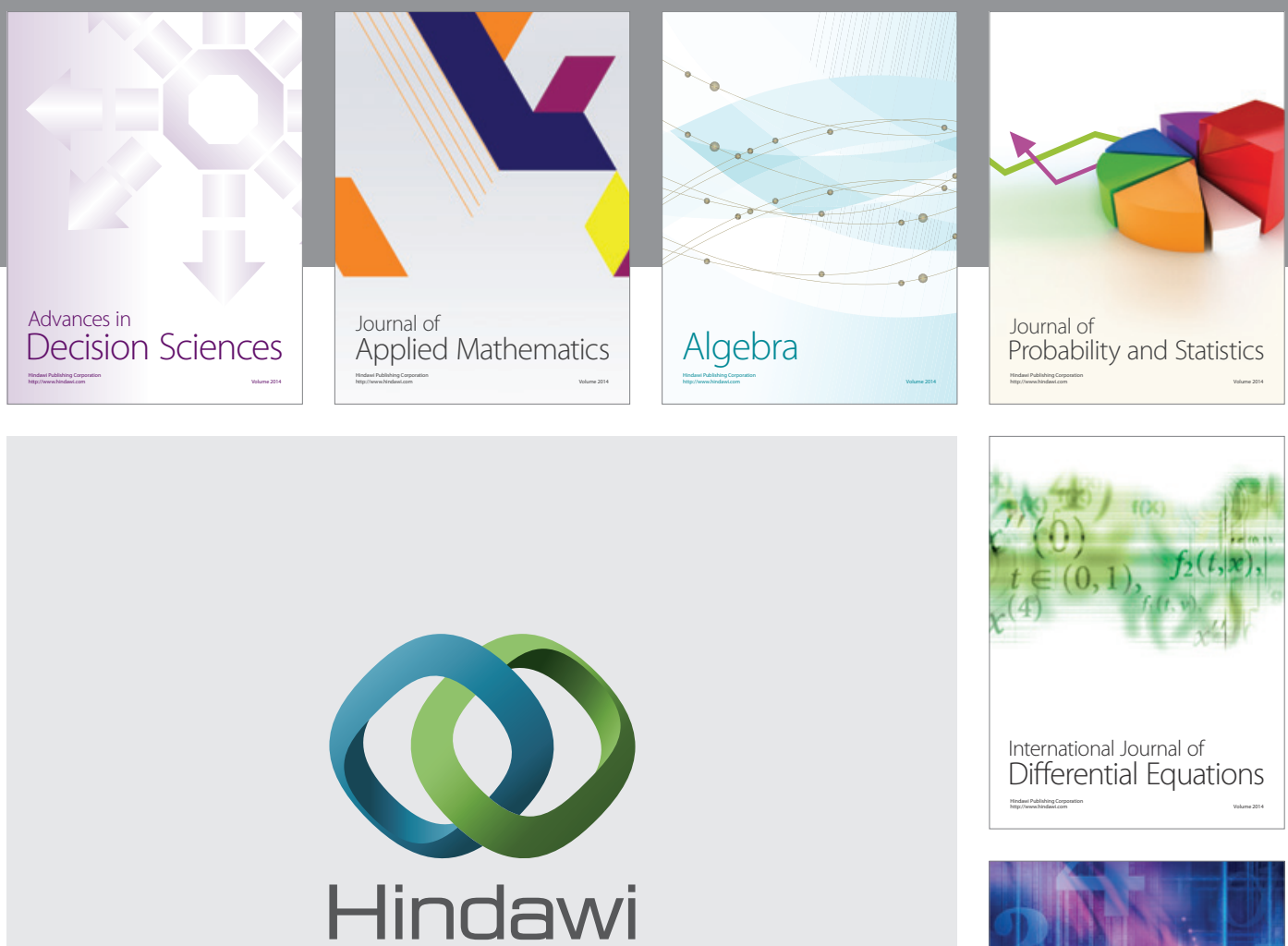

Submit your manuscripts at http://www.hindawi.com
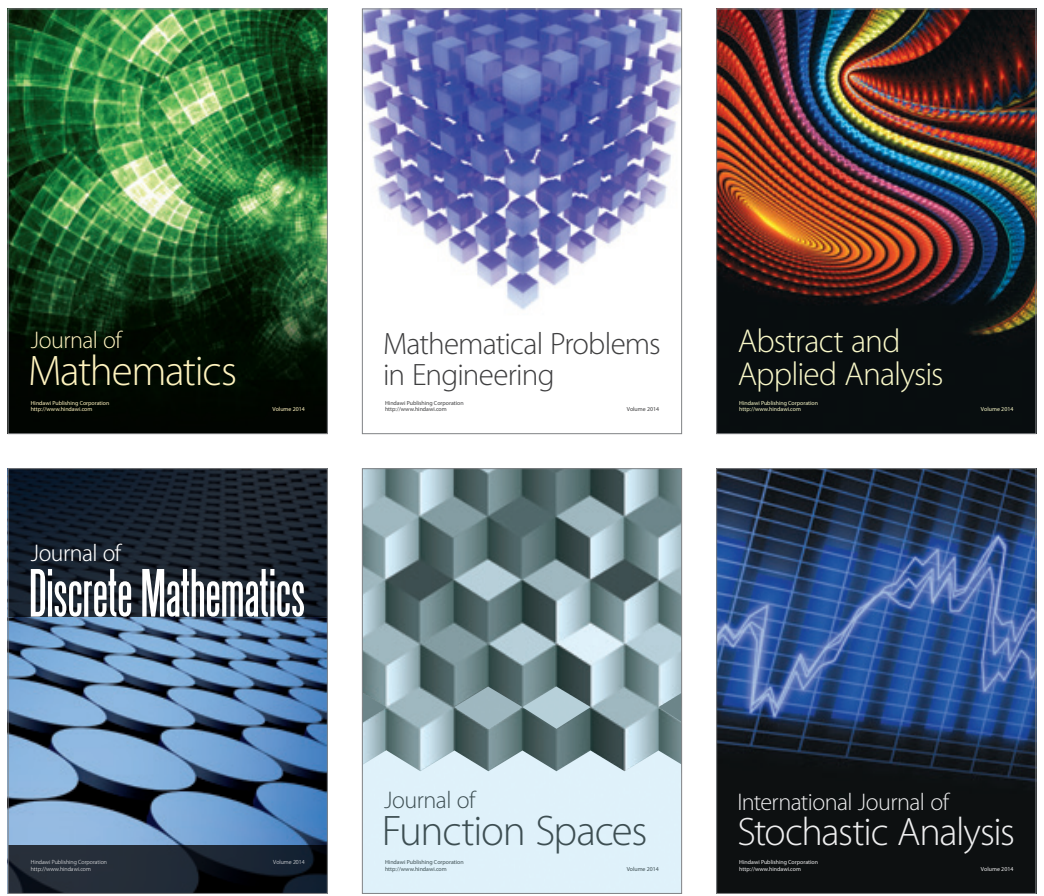

Journal of

Function Spaces

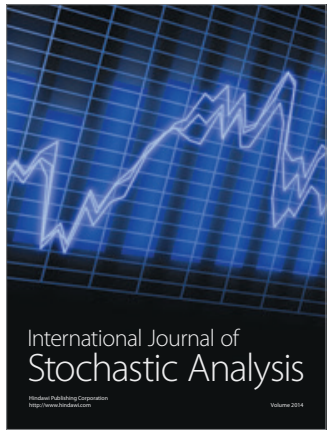

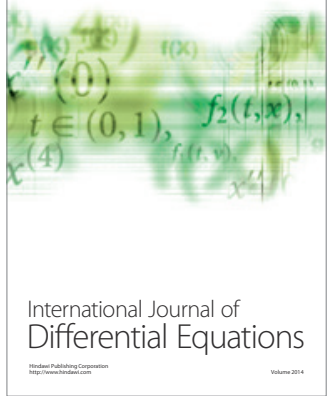
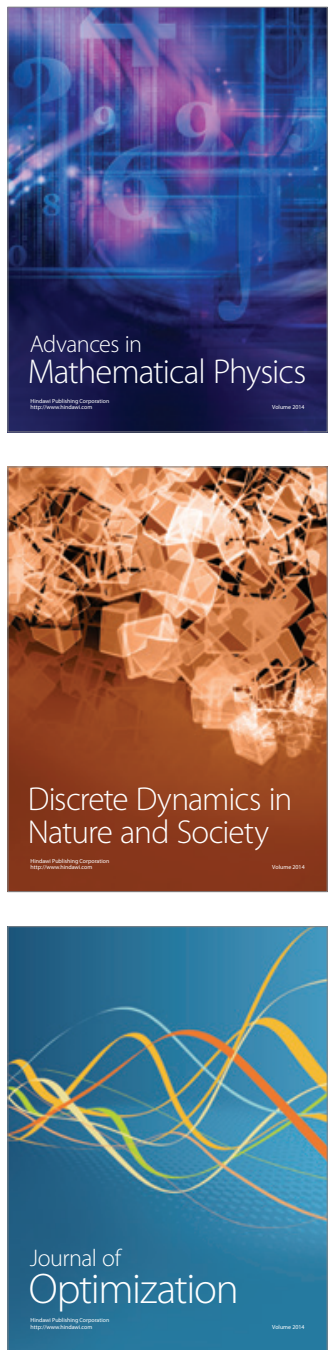